\title{
Legitimität im europäischen Mehrebenensystem
}

\author{
Fritz W. Scharpf
}

Zusammenfasssung: Um gleichzeitig effektiv und liberal sein zu können, ist staatliche Herrschaft auf freiwillige Folgebereitschaft angewiesen - die ihrerseits der Unterstützung durch sozial geteilte Legitimitäts-Überzeugungen bedarf. In den demokratischen Verfassungsstaaten des Westens werden solche Überzeugungen aus den unterschiedlichen, aber komplementär zusammenwirkenden Traditionen der „republikanischen“ und der „liberalen“ politischen Philosophie hergeleitet. An diesen Kriterien gemessen erscheint die Europäische Union - wenn man sie für sich betrachtet - als eine „liberale“ politische Ordnung, der jedoch alle „republikanischen“ Legitimitätsmerkmale fehlen. Aber eine solche Sichtweise, die auch die derzeitige Diskussion über ein „europäisches Demokratiedefizit" bestimmt, verkennt den Mehrebenencharakter des europäischen Gemeinwesens. In ihm sind es die Mitgliedstaaten, die Entscheidungen der Union gegenüber den eigenen Bürgern durchsetzen und auch legitimieren müssen, während es für die Union ihrerseits auf die freiwillige Folgebereitschaft ihrer Mitgliedstaaten ankommt. Dabei werden diese jedoch durch die normativen Grundlagen ihrer eigenen Legitimität begrenzt. Politische Entscheidungen auf europäischer Ebene setzen breiten Konsens voraus, und die Regierungen sollten sie deshalb auch gegenüber den eigenen Bürgern in „kommunikativen Diskursen“ vertreten und dafür die politische Verantwortung übernehmen können. Dies gilt jedoch nicht notwendigerweise auch für Entscheidungen der europäischen Politik, die im nicht-politischen Modus ohne Beteiligung des Rates und des Parlaments vom Europäischen Gerichtshof bestimmt werden. Mit der gegenwärtigen Radikalisierung seines „liberalen“ Programms der Liberalisierung und Deregulierung des nationalen Rechts könnte der Gerichtshof in der Tat die „republikanischen“ Grundlagen der mitgliedstaatlichen Legitimität unterminieren. In diesem Falle könnte die Union sich nicht länger auf die Folgebereitschaft ihrer Mitgliedstaaten verlassen. Um diese Gefahr für die europäische Integration zu vermeiden, sollte eine stärkere politische Kontrolle der richterlichen Rechtsetzung erwogen werden.

Schlüsselwörter: Legitimität · Liberalismus · Republikanismus · Europäische Union (EU) · Europäischer Gerichtshof (EuGH)

\section{(c) VS-Verlag 2009}

Dieser Beitrag hat sehr von Diskussionen am European University Institut (EUI), Florenz, und an der Bremen International Graduate School of Social Sciences (BIGSSS) sowie von den Kommentaren von Martin Höpner am Max-Planck-Institut für Gesellschaftsforschung profitiert. Wie bei allen meinen neuen Arbeiten war die Mitarbeit von Frau Ines Klughardt auch hier von unschätzbarem Wert.

Prof. Dr. F. W. Scharpf $(\bowtie)$

Max-Planck-Institut für Gesellschaftsforschung

Paulstr. 3, 50676 Köln, Deutschland

E-Mail: scharpf@mpifg.de

\section{MPIfG Journal Article}

Fritz W. Scharpf: Legitimität im europäischen Mehrebenensystem. In: Leviathan 37(2), 244 - 280 (2009). Nomos

The original publication is available at the publisher's web site: http://dx.doi.org/10.1007/s11578-009-0016-7

The MPIfG Journal Articles series features articles by MPIfG researchers and visiting scholars published in peer-reviewed journals. Max Planck Institute for the Study of Societies (MPIfG) Cologne | www.mpifg.de 


\title{
Legitimacy in the multilevel European polity
}

\begin{abstract}
In order to be simultaneously effective and liberal, governments must normally be able to count on voluntary compliance - which, in turn, depends on the support of socially shared legitimacy beliefs. In Western constitutional democracies, such beliefs are derived from the distinct but coexistent traditions of "republican" and "liberal" political philosophy. When judged by these criteria, the European Union - if considered by itself - appears as a thoroughly liberal polity which, however, lacks all republican credentials. But this view (which seems to structure the debates about the "European democratic deficit") ignores the multilevel nature of the European polity, where the compliance of citizens is requested, and needs to be legitimated, by member states - whereas the Union appears as a "government of governments" which is entirely dependent on the voluntary compliance of its member states. What matters primarily, therefore, is the compliance-legitimacy relationship between the Union and its member states - which, however, is normatively constrained by the basic compliance-legitimacy relationship between member governments and their constituents. Given the high consensus requirements of European legislation, member governments could and should be able to assume political responsibility for European policies in which they had a voice, and to justify them in "communicative discourses" in the national public space. This is not necessarily true of "non-political" policy choices imposed by the European Court of Justice. By enforcing its "liberal" program of liberalization and deregulation, the ECJ may presently be undermining the "republican" bases of member-state legitimacy. Where this is the case, open non-compliance is a present danger, and political controls of judicial legislation may be called for.
\end{abstract}

Keywords: Legitimacy · Liberalism · Republicanism · European Union (EU) · European Court of Justice (ECJ)

\section{Legitimität}

Nach meinem Verständnis muss jede Diskussion über die Legitimität staatlicher Herrschaft von einer funktionalen Perspektive ausgehen: Sozial geteilte Legitimitäts-Überzeugungen stützen die freiwillige Folgebereitschaft derer, denen die Befolgung unerwünschter staatlicher Regeln oder Entscheidungen zugemutet wird (Easton 1965, S. 278-310; Beetham 1991; Scharpf 1999; Höffe 2002, S. 40). Indem solche Überzeugungen den „Konsens der Verlierer" (Anderson et al. 2005) sozial entlasten und rechtfertigen, verringern sie die Notwendigkeit (und die Kosten) von Kontrollen und Sanktionen, die sonst erforderlich wären, um regelkonformes Verhalten zu erzwingen. ${ }^{1}$ Wenn staatliche Herrschaft zugleich effektiv und liberal sein soll, erfordert sie also Legitimität als funktionale Voraussetzung. Ausgehend von dieser funktionalen Perspektive könnte die weiterführende Arbeit entweder die tatsächliche Folgebereitschaft der Bürger und deren Zusammenhang mit empirisch ermittelbaren Legitimationsvorstellungen untersuchen. Oder sie kann in normativer Absicht fragen, für welche Legitimationsargumente sich im Rahmen der westlichen poli-

1 Die funktionale Bedeutung des Legitimitätsglaubens ist eher eine Variable als eine Konstante. Der Bedarf wächst mit der Schwere der zugemuteten Opfer und er vermindert sich, wenn Ausweichmöglichkeiten zugelassen werden - z.B. wenn die Wartelisten in einem nationalen Gesundheitssystem durch die Inanspruchnahme ausländischer Anbieter umgangen werden können (Martinsen 2009). 
tischen Tradition gute Gründe anführen lassen. Hier will ich mich im Folgenden der normativen Diskussion zuwenden.

\subsection{Republikanische und liberale Legitimationsdiskurse}

Normative Diskurse in westlichen konstitutionellen Demokratien sind von zwei Traditionen politischer Philosophie geprägt, die man in Anlehnung an die anglo-amerikanische Diskussion als ,republikanisch“ und als „liberal“" bezeichnen kann (Bellamy 2006). ${ }^{2}$ Auch wenn manche Autoren zu beiden Traditionen einen Beitrag geleistet haben, sind ihre historischen Ursprünge, Prämissen und die ihre Schlussfolgerungen bestimmende generative Logik doch klar unterscheidbar.

Die republikanische Tradition geht auf Aristoteles zurück. Für ihn hat das Gemeinwesen Vorrang vor dem Individuum, weil es die Entwicklung menschlicher Fähigkeiten erst ermöglicht. ${ }^{3}$ Dann kommt aber alles darauf an, dass die unverzichtbare Regierungsgewalt ausschließlich zur Förderung des Gemeinwohls eingesetzt wird. Jede Regierungsform braucht deshalb institutionelle Vorkehrungen gegen die prinzipiell unsichere „Tugendhaftigkeit" der Herrschenden, die andernfalls ihre Autorität zum eigenen Vorteil einsetzen könnten. Die Sorge für das Gemeinwohl und dessen institutionelle Sicherung prägte später auch die politische Philosophie des republikanischen Roms (Cicero 1995), und sie lebte in der florentinischen Renaissance in den Discorsi Machiavellis (1966, orig. 1531) wieder auf. Von dort führt ein Strang der republikanischen Tradition über die ,neurömischen“ Theoretiker der Englischen Revolution zu den politischen Idealen der Selbstregierung des Volkes in der Amerikanischen Revolution (Pocock 1975; Skinner 1998; Dahl 1989, Kap. 2) und zu den heutigen Vertretern einer ,kommunitaristischen“ Demokratietheorie (Pitkin 1981; MacIntyre 1984, 1988; Pateman 1985; Michelman 1989; Taylor 1992; vgl. Habermas 1992, S. 324-248).

Der andere Strang führt zum radikalen Egalitarismus des Rousseauschen Contrat Social, der das politische Denken der Französischen Revolution prägte und auch die weitere Entwicklung der Demokratietheorie auf dem europäischen Kontinent stark beeinflusst hat. Mit dem klassischen Erbe teilt Rousseau das Primat des Gemeinwesens und des Gemeinwohls, das er durch das Postulat der gleichberechtigten Teilnahme aller Bürger an Prozessen der kollektiven Selbstbestimmung ergänzt. ${ }^{4}$ Aber für ihn wie für Aristoteles wird die „Tugendhaftigkeit" der Herrschenden zum kritischen Problem. In der Theorie soll es durch die Transformation der eigennützigen volonté des tous zur gemeinwohlorientierten volonté générale überwunden werden - eine Lösung, die Rousseau selbst nur für sehr kleine und homogene Gemeinschaften als praktikabel ansah. In der konstituti-

2 Bezogen auf die amerikanische Tradition unterscheidet Robert Dahl (1989, S. 26) zwischen einem ,aristokratischen Republikanismus“ (der die Notwendigkeit betont, den Einfluss von Bevölkerungsmehrheiten durch checks and balances zu begrenzen) und einem ,radikalen Republikanismus" (der die demokratische Gleichheit betont). Im Gegensatz zur liberalen Tradition legen jedoch beide Varianten den Schwerpunkt auf die kollektive Selbstbestimmung statt auf individuelle Freiheiten.

3 Aristoteles 1989, Erstes Buch, 1253a.

4 Rousseau 1959 [1762], 1. Buch, 6. Kapitel; 2. Buch, 1. und 4. Kapitel. 
onellen Praxis wurde dieses Grundproblem des demokratischen Republikanismus dann durch die „Erfindung“ der repräsentativen parlamentarischen Demokratie entschärft, die den institutionellen Rahmen der mittelalterlichen Ständevertretung mit den Forderungen der egalitären Demokratie verband (Dahl 1989, S. 28-30). Die Gemeinwohlorientierung der Repräsentanten soll hier einerseits durch die „Deliberation“ der Abgeordneten und der Bürger im gemeinsamen öffentlichen Raum (Habermas 1962; Elster 1998) gesichert werden, und andererseits durch die institutionalisierte Verantwortlichkeit der Repräsentanten gegenüber der Gesamtheit der Bürger - wobei der egalitäre Anspruch des demokratischen Republikanismus in der konstitutiven Bedeutung des allgemeinen und gleichen Wahlrechts seinen stärksten Ausdruck findet.

Verglichen mit dem Republikanismus ist die „liberale“ Tradition wesentlich jünger; sie geht nicht auf die griechisch-römische Antike zurück, sondern auf die Philosophie der Aufklärung in der frühen Neuzeit und insbesondere auf Thomas Hobbes (1986 [1651]). Im Liberalismus hat das Individuum Vorrang vor dem Gemeinwesen. Der Staat rechtfertigt sich durch die Notwendigkeit des Schutzes individueller Interessen, und die individuelle Selbstbestimmung tritt an die Stelle der kollektiven Selbstregierung. Sobald die Sicherheit von Leben, Freiheit und Eigentumsrechten der Bürger gewährleistet ist, bedarf es strikter Beschränkungen der Staatsgewalt, um den Grundwert der ,negativen Freiheit““ zu schützen, die - in der Tradition von John Locke, Adam Smith und John Stuart Mill - als ,freedom of pursuing our own good in our own way“ (Berlin 1958, S. 11), verstanden werden soll.

Wo die Notwendigkeit der Staatsgewalt nicht geleugnet werden kann, wird die individuelle Freiheit am besten durch einstimmig zu treffende Entscheidungen gewährleistet (Buchanan u. Tullock 1962) - oder durch die Institutionalisierung von checks and balances in Verfassungen mit multiplen Vetopositionen und mit pluralistischen Strukturen der Interessenvermittlung (Dahl 1967). Jedenfalls sollen Entscheidungen soweit überhaupt möglich auf den Konsens der betroffenen Interessen gegründet werden statt auf das Votum demokratisch ermächtigter Mehrheiten.

Im deutschen Zweig der europäischen Aufklärung gründete Immanuel Kant dagegen die individualistische Position nicht auf das Eigeninteresse, sondern auf die moralische Autonomie und Vernunft des Menschen. Weil Individuen zugleich frei und verpflichtet sind, ihrer eigenen Vernunft zu folgen, werden sie einsehen, dass ihre Freiheit durch die gleiche Freiheit aller anderen beschränkt wird. Ihr vernünftiges und moralisches Handeln muss deshalb dem „kategorischen Imperativ“ gehorchen (Kant 1961 [1785]). Aber da der Mensch nun einmal kein reines Vernunftwesen, sondern ,aus krummem Holz geschnitzt“ ist, genügt in der Praxis der moralische Imperativ allein nicht, um die wechselseitige Vereinbarkeit frei gewählter Handlungen zu gewährleisten. Deswegen sind allgemeine Gesetze notwendig, deren Nichtbefolgung von der Staatsgewalt wirksam sanktioniert wird. Sie werden die Gesellschaft dem Zustand der universellen Freiheit näher bringen, wenn sie jeweils Regeln aufstellen, denen alle Betroffenen in ihrer Eigenschaft als autonom und vernünftig Handelnde zustimmen könnten (Kant 1966 [1792], 1992 [1793]). Wie Isaiah Berlin (1958, S. 29-39) jedoch zeigt, könnte dieses Kriterium der potentiellen Konsensfähigkeit auch einen sehr freiheitsbeschränkenden regulativen Staat rechtfertigen - insbesondere wenn die Definition der „Deliberation“ sachverständiger und politisch unabhängiger Agenturen oder Gerichte überlassen bleibt (Somek 2008). Mit anderen 
Worten: Ebenso wie der auf die volonté générale gegründete Republikanismus Rousseaus kann auch der auf den kategorischen Imperativ gegründete Liberalismus Kants durchaus zur Rechtfertigung von Gesetzen verwandt werden, die sich sehr weit von den empirisch feststellbaren Präferenzen real existierender Staatsbürger entfernen.

\subsection{Konstitutionelle Demokratien - und die EU?}

Dieser Überblick überzeichnet gewiss die Unterschiede zwischen den beiden Traditionen der westlichen politischen Philosophie. Eine ausführlichere Darstellung müsste nuancierter und differenzierter ausfallen. Wichtig ist hier jedoch die Einsicht, dass die Legitimität der westlichen Demokratien auf normativen Argumenten beruht, die sich gleichermaßen auf beide Traditionen berufen. Alle demokratischen Verfassungsstaaten sind „liberal“ in dem Sinne, dass die Staatsgewalt konstitutionell beschränkt wird, dass die Menschenrechte geschützt sind und dass die betroffenen Interessen Zugang zu politischen Entscheidungsprozessen finden. Zugleich sind sie alle auch „republikanisch“ in dem Sinne, dass es sich um repräsentative Demokratien handelt, wo die Staatsgewalt durch periodische, allgemeine, freie und gleiche Wahlen erlangt und entzogen wird, wo politische Entscheidungen aus öffentlichen Debatten und dem Wettbewerb politischer Parteien hervorgehen und wo Institutionen, die sich nicht unmittelbar politisch verantworten müssen, doch im Schatten demokratischer Mehrheiten oder wenigstens einer demokratischen pouvoir constituant agieren. Mit anderen Worten, die Institutionen des demokratischen Verfassungsstaates gründen sich gleichzeitig auf republikanische und liberale Prinzipien. Diese beschränken, ergänzen und verstärken sich gegenseitig in der politischen Praxis westlicher Demokratien (Bellamy 2006). In gewissem Sinne kann man sie als Gegengifte gegen die charakteristische Perversion der jeweils anderen Seite verstehen - wenn der republikanische Kollektivismus durch den liberalen Schutz individueller Freiheiten gemäßigt und der libertäre Egoismus durch Institutionen der kollektiven Selbstbestimmung beschränkt wird.

Gewiss variieren die jeweils gewählten Kombinationen, und die Unterschiede zwischen den demokratischen Verfassungsstaaten sind durchaus von Bedeutung. Republikanische Politik wird in Einheitsstaaten erleichtert und durch föderale Verfassungen behindert, Individualinteressen genießen weniger Schutz, wo die Verfassung die parlamentarische Souveränität betont, und der konsensabhängige Pluralismus ist in den Vereinigten Staaten oder in der Schweiz stärker als in Großbritannien, in Neuseeland oder in Frankreich. ${ }^{5}$ Die Bedeutung dieser Unterschiede schwindet jedoch, wenn wir nun statt der demokratischen Nationalstaaten die Europäische Union betrachten. Für sich genommen und unter den zuvor genannten Kriterien erscheint die Union als Extremfall eines den liberalen Prinzipien verpflichteten Gemeinwesens, das zugleich aller republikanischen Legitimitätsmerkmale entbehrt.

5 Richard Münch (2008a, Kap. 4), der eher die „Semantik“ nationaler normativer Diskussionen betrachtet als Institutionen und Praktiken, assoziiert Frankreich mit Republikanismus und Großbritannien mit Liberalismus. Er sieht beide jedoch als Manifestationen eines gemeinsamen europäischen Engagements für „moralischen Universalismus und ethischen Individualismus“, das die europäische Transformation der nationalen Gesellschaften vorantreibe. 
Der Liberalismus der EU zeigt sich am deutlichsten in dem Vorrang, den sie dem Schutz individueller Rechte einräumt und in den institutionellen Hindernissen, die mehrheitlichem politischem Handeln entgegenstehen. So ist der Europäische Gerichtshof (EuGH) unabhängiger von der Politik als das Verfassungsgericht in irgendeinem demokratischen Staat. Und dieses Gericht hat von Anfang an die im EWG-Vertrag vereinbarte Herstellung eines europaweiten Marktes als einen Satz von einklagbaren Rechten der Individuen und Unternehmen interpretiert. Im gleichen Sinne wurde der politisch rudimentäre Status der Unionsbürgerschaft umgedeutet in Individualrechte, die EU-Ausländern Zugang zu den Sozialleistungen und öffentlichen Dienstleistungen aller Mitgliedstaaten verschaffen (Wollenschläger 2007). Überdies hat der EuGH auch begonnen, die allgemeinen Menschenrechte zu schützen, und mit der Einbeziehung der Charta der Grundrechte in den Europäischen Verfassungsvertrag wird der Schutz individueller Rechte noch erweitert.

Gleichzeitig hemmen sehr hohe Konsenserfordernisse das kollektive politische Handeln der Union, und die Input-Struktur der politischen Entscheidungsprozesse könnte nicht pluralistischer und weniger majoritär sein. Die Kommission selbst, die ein Monopol auf legislative Initiativen hat, verfügt über eine ausgedehnte Infrastruktur von Ausschüssen und Expertengruppen, zu denen eine Vielzahl organisierter Interessengruppen Zugang haben. Außerdem haben durch den Ministerrat, in dem bei der Abstimmung über alle Gesetze mindestens eine qualifizierte Mehrheit erforderlich ist, alle Interessengruppen mit Zugang zu den zuständigen nationalen Ministerien auch Zugang zur europäischen Ebene. Schließlich rühmt sich das Europäische Parlament, dessen Rolle im Gesetzgebungsverfahren durch die jüngsten Vertragsrevisionen erheblich erweitert wurde, auch jene nichtorganisierten Interessen zu vertreten, die in der Kommission und im Rat vielleicht ignoriert wurden. Kurz, die europäische Gesetzgebung bietet den organisierten Interessen eine Fülle von Zugangschancen und die hohen Konsensschwellen wirken als effektiver Minderheitenschutz in der europäischen Gesetzgebung. ${ }^{6}$

Dem liberalen Modell entspricht auf der Output-Seite die Effektivität der EU als von politischen Mehrheiten unabhängiger ,regulatory state“ (Majone 1996, 1998). Die Geldpolitik der Europäischen Zentralbank ist vollständig gegen politische Interventionen geschützt. Und bei der Entwicklung eines europäischen Wettbewerbsregimes hat auch die Kommission weitgehende politische Unabhängigkeit erlangt. Im Prinzip gilt dies jedoch ebenso für die Rolle der Kommission und des Gerichts bei der Verfolgung von Vertragsverletzungen der Mitgliedstaaten auf allen anderen Rechtsgebieten.

Aber während die EU dem liberalen Standard durchaus entspricht, würde sie an den Kriterien einer republikanischen Demokratie eindeutig scheitern. In der Output-Dimension verhindern die Konsenserfordernisse der EU-Gesetzgebung wirksames kollektives Handeln bei vielen Problemen, welche die Mitgliedstaaten national nicht bewältigen können. Die notorische Unfähigkeit der EU, den Wettbewerb über Steuern auf Unternehmensgewinne und Kapitaleinkünfte zu regulieren, ist dafür nur ein Beispiel (Ganghof u. Genschel 2008a,b). Schlimmer noch, dieselben Entscheidungsregeln sind auch für eine

6 Konsens ist natürlich auch das Prinzip der „,new modes of governance“, die eine Koordinierung durch „Soft law“, Benchmarking“ und institutionelles Lernen auf Gebieten anstreben, auf denen der EU Gesetzgebungskompetenzen fehlen (Héritier 2003; Héritier u. Lehmkuhl 2008; KohlerKoch u. Rittberger 2006). 
extrem konservative Tendenz der EU-Politik verantwortlich. Neue Gesetze mögen auf einem breiten Konsens basieren, aber sobald sie einmal beschlossen sind, können sie nicht mehr aufgehoben oder an veränderte Umstände oder Präferenzen angepasst werden, wenn entweder die Kommission oder einige Mitgliedstaaten an der bestehenden Regel festhalten wollen. Ist die betreffende Regel aus der gerichtlichen Interpretation der Verträge abgeleitet, wäre sie überdies nur durch eine Vertragsänderung zu korrigieren, welche einstimmig von allen Regierungen beschlossen und durch die Parlamente oder durch Volksentscheid in sämtlichen Mitgliedstaaten ratifiziert werden müsste. Mit anderen Worten, sobald ein EU-Gesetz beschlossen ist, ist der acquis fast irreversibel, selbst wenn er im Laufe der Zeit immer weniger dem Gemeinwohl entspricht.

Die hohen Konsenserfordernisse können allerdings kaum vermindert werden, so lange es den Völkern der 27 Mitgliedstaaten an einer kollektiven Identität mangelt, die europaweite Mehrheitsentscheidungen legitimieren könnte. Und selbst wenn die EU-Bürger (vielleicht als Reaktion auf äußere Herausforderungen) ein starkes Gefühl gemeinsamer Solidarität entwickelten, und wenn ihnen die Zugehörigkeit zur Union wichtiger würde als die zum eigenen Nationalstaat, so fehlten ihnen derzeit doch alle gesellschaftlichen und institutionellen Voraussetzungen der input-orientierten Demokratie: Es gibt keine europaweiten Kommunikationsmedien und politischen Debatten, keine europaweiten politischen Parteien, keinen europaweiten, auf wichtige europapolitische Entscheidungen konzentrierten Parteienwettbewerb und keine politisch verantwortliche europäische Regierung, die antizipierend auf das mögliche Votum der Wähler in egalitären, europaweiten Wahlen reagieren müsste. Es gibt zwar keinen theoretischen Grund, diese Defizite als unveränderlich zu betrachten, aber gegenwärtig kann die EU keinen Anspruch auf input-orientierte republikanische Legitimation erheben.

Mögen diese stilisierten Diagnosen auch etwas überzeichnet sein, so liefern sie doch eine auf den ersten Blick plausible Interpretation der derzeitigen Auseinandersetzung über das sogenannte ,demokratische Defizit“. Autoren und politische Akteure, die von einem ,liberalen“ Verständnis normativer politischer Theorie ausgehen, werden die Legitimität der Europäischen Union mit dem Schutz individueller Rechte, der pluralistischen Offenheit politischer Prozesse, den konsensualen Entscheidungsregeln und mit der Wirksamkeit ihrer regulativen Politik begründen sehen (Moravcsik 1998, 2002). Im Gegensatz dazu werden Autoren und politische Akteure, welche die EU aus einer „republikanischen“ Perspektive beurteilen, auf Defizite in der Output-Dimension und die Vernachlässigung redistributiver Politikziele verweisen. Die gravierendere Kritik betrifft jedoch Defizite in der Input-Dimension - das Fehlen eines gemeinsamen „öffentlichen Raums“ und europaweiter politischer Debatten, den mangelnden Parteienwettbewerb und die fehlende politische Verantwortlichkeit der Regierenden (Greven 2000; Harlow 2002; Follesdal u. Hix 2006; Hix 2008). Einige der Kritiker glauben zwar, dass diese Mängel in absehbarer Zeit durch institutionelle Reformen und die Mobilisierungsstrategien europäischer Parteien überwunden werden können. Sie unterschätzen aber wohl die Risiken politischer Mobilisierung und Konfrontation in einem institutionellen Rahmen, der - angesichts des Fehlens einer starken kollektiven Identität - noch immer nur Entscheidungen im breiten Konsens zulässt (Bartolini 2005, 2008). 


\section{Legitimität in Mehrebenen-Gemeinwesen}

Auf jeden Fall genügt die Europäische Union in ihrer gegenwärtigen Form den republikanischen Kriterien demokratischer Legitimität so wenig, dass sie nicht von der wechselseitigen Verstärkung liberaler und republikanischer Prinzipien profitieren kann, auf der die Legitimität konstitutioneller Demokratien auf nationaler Ebene beruht (Preuss 1999). Aber kommt es darauf überhaupt an, wenn man anerkennt, dass die EU nicht als ein selbständiges Gemeinwesen existiert? In der Zwei-Ebenen-Konstellation des europäischen politischen Systems wird ja von den Mitgliedstaaten in der Tat erwartet, dass sie im vollen Umfang sowohl den liberalen als auch den republikanischen Legitimitätskriterien gerecht werden. Aber dann müsste man doch auch fragen, welche normativen Folgerungen aus dieser Tatsache für die Anforderungen an die europäische Ebene und für die Einschätzung der Gesamt-Legitimität des europäischen Zwei-Ebenen-Systems zu ziehen sind.

Bei der Suche nach einer Antwort lohnt es sich, die Herrschaftsbeziehung und die Legitimationsbeziehung zwischen Bürgern und Staatsgewalt politischen Systemen mit unterschiedlicher institutioneller Architektur miteinander zu vergleichen. In einem Einheitsstaat sind diese Beziehungen kongruent. Die Zentralregierung erlässt die Gesetze und ihre Verwaltungsorgane fordern den Gehorsam der Bürger. Im Gegenzug verbindet auch die Legitimitätsbeziehung die Bürger unmittelbar mit der Zentralregierung, deren Politik in der nationalen Öffentlichkeit zur Diskussion steht, die sich dem nationalen Parteienwettbewerb stellen muss, und die vom Wähler in allgemeinen Wahlen sanktioniert werden kann. Kongruenz kann auch in Zwei-Ebenen-Gemeinwesen erreicht werden, wenn deren institutionelle Architektur mit dem Modell des „dualen Föderalismus“ übereinstimmt. Hier hat jede Regierungsebene ihre eigene Domäne autonomer Gesetzgebung, ihre eigenen Strukturen des Gesetzesvollzugs und ihre eigene politische Infrastruktur von Parteien, Wahlkämpfen und Medien, in der ihre politische Verantwortlichkeit gegenüber den eigenen Wählern diskutiert wird.

Komplizierter sind die Dinge im deutschen Modell eines „unitarischen Bundesstaats“, wo die meisten Gesetzgebungsbefugnisse beim Bund liegen, dessen Gesetze dann jedoch von den Ländern vollzogen werden. Von den Landesbehörden wird erwartet, dass sie sich an die Bundesgesetze halten, und von den Bürgern wird erwartet, dass sie den Anordnungen der Landesbehörden folgen, gleich ob diese sich auf Bundes- oder Landesrecht stützen. In der unitarischen politischen Kultur des deutschen Bundesstaates wird die zweistufige Herrschaftsbeziehung jedoch nicht zu einem Legitimationsproblem. Politische Diskussionen finden in bundesweiten Medien und in einem bundesweiten öffentlichen Raum statt. Die Aufmerksamkeit der Öffentlichkeit konzentriert sich auf die Bundespolitik; und selbst bei Landtagswahlen tragen die Parteien in erster Linie bundespolitische Kontroversen aus und interpretieren die Ergebnisse als Plebiszit über die Politik der jeweiligen Bundesregierung - das ja über die parteipolitische Zusammensetzung des Bundesrates auch unmittelbare Rückwirkungen auf diese haben kann (Burkhart 2008; Scharpf 2009). Während also die staatliche Herrschaft den Bürgern in den Ländern entgegentritt, verbindet die Legitimationsbeziehung die Bürger mit der Bundespolitik, die in Bundes- wie in Landtagswahlen zur Verantwortung gezogen werden kann.

Die Zwei-Ebenen-Konstellation zwischen der Europäischen Union und ihren Mitgliedstaaten hat mit dem deutschen Föderalismus einige wichtige strukturelle Merkmale 


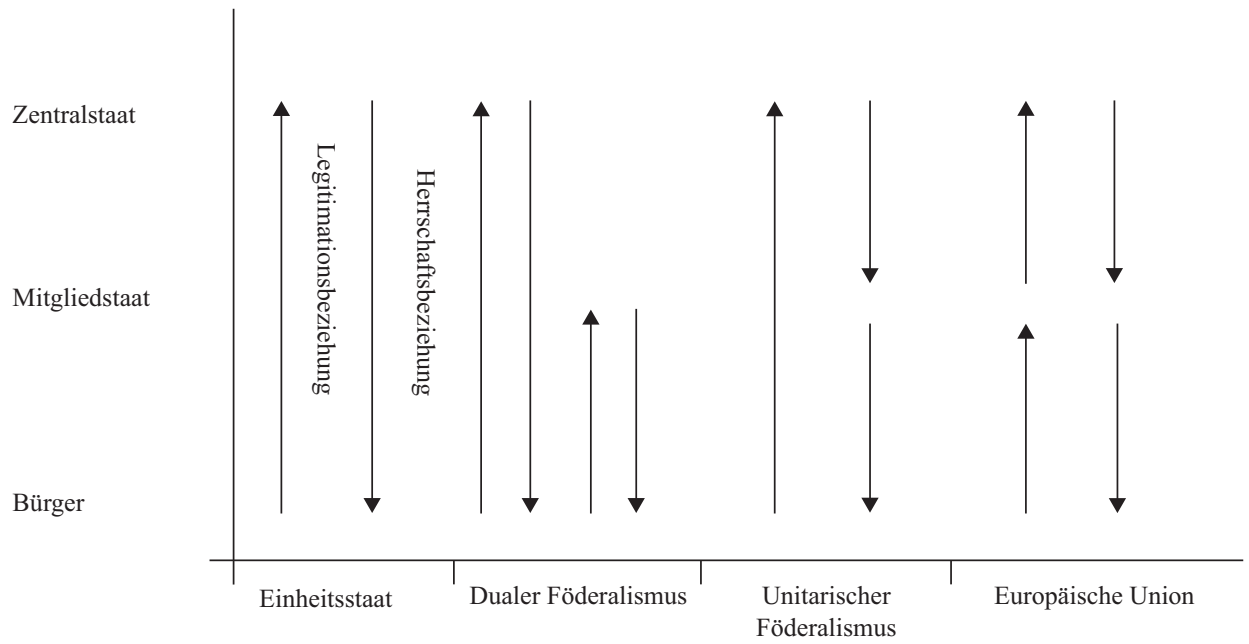

Abb. 1: Folgebereitschaft und Legitimation in Mehrebenen-Regierungen

gemeinsam (Scharpf 1988). Aber im Kontext einer Diskussion über politische Legitimität sind die Unterschiede gewichtiger als die Ähnlichkeiten. Die Union ist im Vergleich zum Bund viel abhängiger von ihren Mitgliedstaaten: Der nationale Gesetzgeber muss die europäischen Richtlinien umsetzen; Verwaltungsorgane und Gerichte der Mitgliedstaaten müssen das europäische Recht vollziehen; und der europäische Haushalt hängt fast vollständig von den Beitragszahlungen der Mitglieder ab. Als Konsequenz erscheint die Herrschaftsbeziehung noch klarer als in Deutschland als ein zweistufiges Verhältnis.

Aus Sicht der Bürger wird Folgebereitschaft ausschließlich von den mitgliedstaatlichen Verwaltungsorganen, Steuerbehörden und Gerichten eingefordert. Und abgesehen von dem Fall, dass die Kommission unmittelbar die Verletzung von Wettbewerbsregeln ahndet, sehen sich selbst Unternehmen niemals direkt mit Vollzugsorganen der Europäischen Union konfrontiert. Die Folgebereitschaft, die aus Sicht der Europäischen Union von Bedeutung ist, ist die Bereitschaft und Fähigkeit der Regierungen ihrer Mitgliedstaaten, die Befolgung von EU-Recht sicherzustellen. Dies ist die „compliance“, die von der Kommission kontrolliert wird und die auch in der Europaforschung einen Schwerpunkt empirischer Untersuchungen bildet (Falkner et al. 2005; Zürn u. Joerges 2005; Börzel u. Dudziak 2007).

Ebenso wie in Deutschland haben wir also in der EU eine zweistufige Herrschaftsbeziehung - zwischen der Union und den Mitgliedstaaten und zwischen diesen und deren Bürgern. Anders als in Deutschland aber haben wir in Europa auch eine zweistufige Legitimationsbeziehung: ${ }^{7}$ Während die Bürger im deutschen Föderalismus ihre Forderungen

7 In der europarechtlichen Literatur und in Kommunikationen der Kommission wird häufig der Schaffung unmittelbar geltender subjektiver Rechte ein die EU legitimierender Effekt zugeschrieben. Ein Problem der Folgebereitschaft, und damit ein Legitimationsproblem, gibt es jedoch in dem hier vertretenen Verständnis nur bei der herrschaftlichen Zumutung unerwünschter Verhaltensregeln. Die Einräumung subjektiver Rechte bedarf für die dadurch Begünstigten kei- 
in der Regel an die Bundespolitik adressieren und diese auch in allfälligen Wahlen zur politischen Verantwortung ziehen, wird die europäische Politik von den Aktionen und Reaktionen der Bürger nicht erreicht. Sie ist weder das Ziel öffentlicher Forderungen, Debatten und des Parteienwettbewerbs, noch kann sie durch Sanktionen der Wähler belohnt oder bestraft werden (Mair 2008). Was die Bürger betrifft, so sind sie durch eine direkte Legitimationsbeziehung nur mit der Regierung ihres Mitgliedstaats verbunden. Und da Wähler nicht verpflichtet sind, fair zu sein - und im Übrigen auch gar nicht die Herkunft der Gesetze kennen können, die sie befolgen sollen -, ist ,die Politik der Schuldvermeidung“ (Weaver 1986) für die Regierungen der Mitgliedstaaten keine politisch nützliche Strategie. Tatsächlich müssen sie die volle Bürde der politischen Verantwortung für die Ausübung ihrer Staatsgewalt tragen, ohne Rücksicht darauf, wie viel das Europarecht zur Unzufriedenheit ihrer Bürger beigetragen haben mag.

Im europäischen Zwei-Ebenen-Gemeinwesen muss deshalb die Union nicht als eine Regierung von Bürgern, sondern als eine Regierung von Regierungen angesehen und als solche auch legitimiert werden. In erster Linie kommt es auf die Bereitschaft und Fähigkeit der Mitgliedstaaten an, das EU-Recht umzusetzen und die politische Verantwortung dafür zu übernehmen. Es erscheint daher völlig angemessen, dass sich die Compliance-Forschung ausschließlich auf die Beziehung zwischen der EU und ihren Mitgliedstaaten konzentriert. Aber wenn das so ist, dann spricht auch nichts dafür, dass man in normativen Diskussionen über die Legitimität der EU diese als ein isoliert existierendes Gemeinwesen behandeln und dass man dabei monistische Begriffe verwenden dürfte, die die Zweistufigkeit der Legitimationsbeziehungen ignorieren. Statt über die Frage eines ,demokratischen Defizits“ im unmittelbaren Verhältnis zwischen der EU und den Bürgern der Mitgliedstaaten zu streiten, sollten wir die legitimierenden Argumente diskutieren, welche den Gehorsam der Mitgliedstaaten gegenüber Anordnungen der EU rechtfertigen können. Und wir sollten die Bedingungen klären, die es den Mitgliedstaaten ermöglichen, diese Folgebereitschaft gegenüber ihren Bürgern zu rechtfertigen.

\section{Was legitimiert die Folgebereitschaft der Mitgliedstaaten?}

Für die Regierungen der Mitgliedstaaten ist die Zugehörigkeit zur Europäischen Union durch deren Beitrag zu Frieden und Demokratie auf dem europäischen Kontinent allemal gerechtfertigt. Weniger deutlich ist dagegen, ob auch die Versprechungen der Wirtschaftsintegration sich erfüllt haben. Auf jeden Fall aber bleibt die Mitgliedschaft für die unmittelbaren Nachbarn der Union weiterhin attraktiv, und ein Austritt aus der Union scheint bisher auch nicht auf der Tagesordnung eines der alten oder der neueren Mitgliedstaaten zu stehen. Aber ebenso wie die Tatsache, dass die meisten Bürger nicht emigrieren wollen, kein ausreichender Beleg für die demokratische Legitimität eines Nationalstaates ist, kann auch eine ganzheitlich positive Einschätzung der Vorteile der Mitgliedschaft für sich allein nicht die Legitimität aller europäischen Verpflichtungen begründen. Wie im demokratischen Nationalstaat kommt es auch hier auf spezifischere Merkmale der Ins-

ner Legitimation, und es ist auch nicht einzusehen, weshalb sie für Dritte ein legitimierendes Argument begründen sollte. 
titutionen und Entscheidungsprozesse an, in denen die an die Mitgliedstaaten adressierten Mandate generiert werden. Dabei erscheint es mir nützlich, grundsätzlich zwischen den ,politischen“ und den ,nichtpolitischen“ Modalitäten der Politikformulierung in der EU zu unterscheiden (Scharpf 2001). Zum politischen Modus zähle ich alle Verfahren, bei denen die Regierungen der Mitgliedstaaten eine Stimme haben - am unmittelbarsten bei Verhandlungen über den EU-Vertrag und in jenen Politikfeldern, in denen noch einstimmige Beschlüsse erforderlich sind. Aber auch dort, wo die Gesetzgebung nach der „Gemeinschaftsmethode“ von einer Initiative der Kommission und der Zustimmung des Europäischen Parlaments abhängt, sichern die Notwendigkeit qualifizierter Mehrheiten im Rat und die Konsens fördernden Verfahren den Einfluss der Regierungen auf das Ergebnis. Im nichtpolitischen Modus europäischer Politikformulierung ist dies nicht der Fall. Weder die Regierungen der Mitgliedstaaten, noch das Europäische Parlament haben eine Stimme, wenn die Europäische Zentralbank den Kurs der Geldpolitik bestimmt, wenn die Kommission gewisse Praktiken der Mitgliedstaaten als Vertragsverletzung verfolgt oder wenn der Europäische Gerichtshof seine Interpretationshoheit nutzt, um den materiellen Gehalt des primären oder sekundären europäischen Gemeinschaftsrechts zu erweitern. Da die Wirkung der auf diesen Wegen beschlossenen Politik die Bedeutung vieler EURichtlinien übertreffen kann, muss auch deren Legitimität explizit erörtert werden.

\subsection{Politische Modi des Politikprozesses}

Für die Regierungen der Mitgliedstaaten, so könnte man meinen, müssen die hohen Konsenserfordernisse der EU-Gesetzgebung deren Input-Legitimität gewährleisten. EU-Verordnungen und Richtlinien können (mit wenigen Ausnahmen - etwa in Art. 86 Abs. 3 EGV) nicht ohne Zustimmung des Rates beschlossen werden. Und auch dort, wo der Rat mit qualifizierter Mehrheit entscheiden kann, sind die Konsens fördernden Praktiken so effektiv, dass politisch wichtige nationale Interessen, die eine Regierung nachdrücklich verteidigt, nur selten überstimmt werden. Aber das bedeutet nicht, dass die EU-Gesetzgebung aus Sicht der Regierungen der Mitgliedstaaten problemlos wäre.

Das offensichtlichste Problem besteht darin, dass eine Mehrheit von Mitgliedstaaten wegen der hohen Konsenserfordernisse oft ${ }^{8}$ daran gehindert wird, ,europäische Lösungen“ für Probleme zu finden, die ihres Erachtens auf der europäischen Ebene gelöst werden sollten und könnten. Aus deren Sicht bleibt deshalb die Output-Legitimität der europä-

8 Oft, aber nicht immer. Es gibt durchaus Politikfelder, wo die EU-Gesetzgebung ,fortschrittlicher" und ,perfektionistischer" zu sein scheint, als man angesichts der politischen Präferenzen der Mitgliedstaaten erwarten würde - z.B. beim Verbraucherschutz, der Arbeitssicherheit oder der Umweltpolitik. Ein Grund dafür kann das starke Engagement „europhiler“ nationaler Vertreter im Sekretariat des Rates und im COREPER sein (Lewis 2005). Aber mindestens eine Ursache kann auch die relative Schwäche der intersektoralen Koordination in der Kommission und im Rat sein. Ambitionierte Fachminister, deren Initiativen zu Hause in interministeriellen Verhandlungen blockiert werden, könnten dann ihre Ziele im zwischenstaatlichen Konsens der Spezialisten im jeweiligen Rat erreichen. Blockaden und Kompromisse auf dem kleinsten gemeinsamen Nenner sollte man also in erster Linie dort erwarten, wo zwischenstaatliche Konflikte im selben Politikfeld auftreten - wie z.B. bei der Steuerharmonisierung, den Arbeitsbeziehungen oder der Sozialpolitik. 
ischen Gesetzgebung systematisch eingeschränkt. Immerhin aber bleiben die betroffenen Regierungen nach dem Scheitern einer neuen europapolitischen Initiative noch in der Lage, das fragliche Problem so gut wie möglich auf nationaler Ebene zu bewältigen. Eine potenziell viel gravierendere Schwierigkeit taucht jedoch auf, wenn die gescheiterte Initiative eine bereits bestehende europäische Regelung hätte reformieren sollen. Diese hat ja nicht nur Vorrang vor existierendem nationalem Recht, sondern sie ,besetzt auch das Feld“" und verhindert so alle künftigen Bemühungen um eine nationale Lösung.

Gleichzeitig wird die existierende europäische Vorschrift aber nun durch dieselben hohen Konsenserfordernisse, die ihre frühere Annahme erschwerten, auch vor europäischen Reforminitiativen geschützt. Sie wird, auch wenn sie ihr Ziel verfehlt oder wenn die Umstände oder die politischen Präferenzen der meisten Mitgliedstaaten sich wesentlich geändert haben, in Kraft bleiben, so lange sie noch entweder von der Kommission (ohne deren Initiative keine Änderung möglich ist) oder von einer kleinen blockierenden Minderheit im Rat verteidigt wird. ${ }^{9}$ Das heißt, die europäische Gesetzgebung ist viel weniger reversibel als nationale Gesetze, die mit einfacher Mehrheit beschlossen, abgeändert und aufgehoben werden können. ${ }^{10}$

\subsection{Nichtpolitische Politikprozesse}

Die Ergebnisse der politischen Gesetzgebung müssen also keineswegs dauerhaft den mehrheitlichen Präferenzen der Mitgliedstaaten entsprechen. Noch weniger gilt dies selbstverständlich für die nichtpolitischen Entscheidungsverfahren, an denen die Regierungen nicht beteiligt sind. Für die Geldpolitik der Europäischen Zentralbank legte der Maastrichter Vertrag den absoluten Vorrang der Preisstabilität vor allen anderen Zielen der Wirtschaftspolitik fest (Art. 105 EG). Und selbst wenn viele Regierungen heute ein flexibleres Mandat vorzögen, könnten sie dies nicht gegen den Einspruch auch nur eines einzelnen Mitgliedstaates beschließen. Und auch wo der Europäische Gerichtshof seine Entscheidungen auf die Kompetenz zur Interpretation und Anwendung des Vertrages (Art. 220 EG) stützt, könnten diese nur auf dem Wege der einstimmigen Vertragsänderung korrigiert werden - und kaum weniger schwierig sind politische Korrekturen einer richterlichen Interpretation europäischer Verordnungen und Richtlinien zu erreichen.

9 Tatsächlich kann der Widerstand gegen eine Reform größer sein als der Widerstand gegen die ursprüngliche Einführung einer Regel. Diese wurde vielleicht durch ein gemeinsames Interesse an einer „europäischen Lösung“ für dringende nationale Probleme begünstigt. Aber wenn dieses Interesse befriedigt ist, können Nutznießer der Status-quo-Regel sich späteren Reformen widersetzen. Das Problem muss für die neuen Mitgliedstaaten besonders akut sein, die durch einen aquis gebunden sind, bei dessen Annahme sie keine Stimme hatten, der vielleicht ihren Bedingungen nicht entspricht und der nicht modifiziert werden kann, um ihren Interessen und Präferenzen besser zu entsprechen.

10 Schon vor über zwei Jahrzehnten sprachen Cappelletti et al. (1985, S. 40) von der ,akuten Gefahr der rechtlichen Obsoleszenz“, die aus „,der Kombination bindender Instrumente und irreversibler Kompetenz der Gemeinschaft, verbunden mit dem immer komplizierteren und langwierigeren Entscheidungsprozess der Gemeinschaft, “ erwächst. Diese Gefahr ist im Laufe der Zeit nicht geringer geworden. 
Die Schwierigkeit einer politischen Revision bestehenden Europarechts erzeugt aber nicht nur eine systematische Asymmetrie zwischen den Verteidigern des Status-quo und den Befürwortern des Wandels, sondern vor allem auch eine Asymmetrie in der PrincipalAgent-Beziehung zwischen denen, die politisch legitimiert sind, europäisches Recht zu setzen, und denen, die dieses Recht anwenden sollen. Da die Rechtsanwendung immer auch eine Interpretation der Regel erfordert, haben die Anwender zwangsläufig eine gewisse Macht, deren Inhalt zu beeinflussen. Und die Reichweite ihrer Macht wird umso größer, je weniger die Gesetzgeber in der Lage sind, Interpretationen zu korrigieren, die von der ursprünglichen Absicht abweichen (Tsebelis 2002). Angesichts der außerordentlichen Schwierigkeit von Änderungen des primären und sekundären Europarechts ist der potentielle Spielraum der richterlichen Rechtsetzung in der Europäischen Union größer als in allen demokratischen Verfassungsstaaten.

Aber warum sollte man in der größeren Reichweite des Richterrechts überhaupt ein Legitimitätsproblem sehen? Wo diese Frage in der politikwissenschaftlichen Europaliteratur überhaupt in Betracht gezogen wird, wird sie mit zwei Rational-Choice-Argumenten verneint, die zeigen sollen, dass eine weite Rechtsetzungskompetenz des Gerichtshofs dem mutmaßlichen Willen, oder jedenfalls dem wohlverstandenen Eigeninteresse der vertragsschließenden Regierungen entsprochen habe und noch entspreche (Garrett 1992, 1995).

Das erste Argument unterstellt, dass die Regierungen beim Abschluss der Verträge eine Konstellation vorhersehen mussten, die dem spieltheoretischen Modell eines (symmetrischen) „N-Personen-Gefangendilemmas“ entsprach: Die vereinbarten FreihandelsRegeln dienten zwar dem gemeinsamen Interesse aller Vertragspartner. Ihre Einhaltung war jedoch nicht gesichert, weil jeder versucht sein würde, sich als Trittbrettfahrer auf Kosten der anderen noch besser zu stellen. Damit aber käme eine Kettenreaktion in Gang, die das vereinbarte Regelwerk zerstören würde. Deshalb sei es aus Sicht der Regierungen vernünftig gewesen, Organe zu schaffen, die nicht unter ihrer direkten politischen Kontrolle stehen, und diese zur Überwachung und Sanktion etwaiger Vertragsverletzungen zu ermächtigen.

Soweit die Annahmen zutreffen, erscheint die Schlussfolgerung in der Tat logisch. Freilich beschränkt sich die Rechtsprechung des Gerichtshofes nicht auf die Kontrolle protektionistischer Free-Rider-Praktiken, sondern erstreckt sich auf eine große Zahl von Politikfeldern, die keineswegs dem symmetrischen Dilemma-Modell entsprechen. Vor allem aber wird das Argument in seiner hier zur Diskussion stehenden Verwendung theoretisch überdehnt. Das Gefangenendilemma rechtfertigt nur die Schaffung politisch unabhängiger Vollzugsbehörden, die die Einhaltung der politisch vereinbarten Regeln überwachen und sanktionieren können. Es liefert jedoch keine analytischen oder normativen Argumente, die begründen könnten, weshalb die Definition der Regeln selbst von den politisch verantwortlichen Organen auf die Rechtsprechung übergehen sollte. ${ }^{11}$

11 Auf ähnliche empirische und theoretische Einwände treffen Argumente, die zeigen sollen, dass der effizienzorientierte „regulative Staat“ einer politischen Legitimation gar nicht bedürfe (Majone 1996). Empirisch gelten sie bestenfalls für einen eng begrenzten Teil der europäischen Politikfelder. Selbst dort setzen die Effizienzargumente jedoch Werturteile über die angestrebten Ziele und die dafür zulässigen Mittel voraus, und auch effizienzorientierte Beschlüsse führen zu 
Diesen Einwand vermeidet ein zweites Argument, das die Rechtsetzungskompetenz des Gerichtshofs durch ein Incomplete-Contracting-Modell (Masking u.Tyrol 1999; Battigalli u. Maggi 2003) rechtfertigen will. Es postuliert, dass vernünftige Vertragsparteien einsehen werden, dass sie nicht alle künftigen Eventualitäten vorhersehen und regeln können. Da sie aber auch die hohen Transaktionskosten dauernder Neuverhandlungen scheuen, werden sie bereit sein, künftige Dispute über die Interpretation des Vertrages durch eine neutrale Instanz entscheiden zu lassen. Das Argument ist jedoch nur plausibel, wenn weitgehend harmonische Interessenkonstellationen vorausgesetzt werden - die etwa dem spieltheoretischen Modell des „Kampfes der Geschlechter“ ähnelt, bei dem alle Beteiligten eine gemeinsame Lösung der Nichteinigung vorziehen, obwohl sie jeweils unterschiedliche Lösungen anstreben (Scharpf 1997, Kap. 6). Unter dieser Annahme lässt sich zwar die Initiativfunktion der Kommission und ihre Rolle als „ehrlicher Makler“ im Prozess der politischen Gesetzgebung begründen, aber das Argument ist keineswegs geeignet, die Legitimität von Richterrecht generell zu stützen.

Zur Erläuterung unterscheide ich zwei Gruppen von Mitgliedstaaten. In der einen entspricht die Wirtschaftsordnung dem - in der Varieties-of-Capitalism-Literatur beschriebenen - Modell einer ,liberalen Marktwirtschaft“ und ihre Regierungen streben deshalb auch ein liberales europäisches Regime an. Die Wirtschaftsordnung in den Ländern der anderen Gruppe entspricht dem Modell der „koordinierten Marktwirtschaft“ und ihre Regierungen wollen auch auf der europäischen Ebene einen regulierten Kapitalismus institutionalisieren (Hall u. Soskice 2001). Auf dem Wege der politischen Gesetzgebung könnte man vielleicht einen europäischen Kompromiss finden, den beide Seiten einem ungeregelten Nebeneinander ihrer Wirtschaftsordnungen vorziehen würden. Aber falls man sich nicht einigt, bliebe es bei dem „Wettbewerb der Systeme“, in dem beide Seiten die komparativen Vorteile ihrer jeweiligen nationalen Regime nutzen können.

Ganz anders fiele das Ergebnis aus, wenn es statt von der politischen von der richterlichen Rechtsetzung bestimmt wird. Diese kann nur in Einzelfallentscheidungen entwickelt werden, in denen es jeweils um die europarechtliche Zulässigkeit einer bestimmten nationalen Regelung geht. Wird diese als Verletzung europäischer Wirtschaftsfreiheiten gewertet, so kann das Gericht sie außer Kraft setzen, ohne dass der betroffene Mitgliedstaat zustimmen müsste. Der Gerichtshof hat jedoch nicht die Möglichkeit, ein gemeinsames europäisches Regime zu schaffen. Er kann nur nationale Regelungen überprüfen, die den freien Verkehr von Waren, Dienstleistungen, Kapital und Personen, die Niederlassungsfreiheit oder den unverfälschten Wettbewerb behindern könnten. In den Grenzen seiner Zuständigkeit hat das Gericht dann nur die Wahl, die angegriffene nationale Regel entweder bestehen zu lassen oder sie aufzuheben. Wenn das Gericht also überhaupt interveniert, dann können seine Urteile nur eine liberalisierende und deregulierende Wirkung haben, die sich einseitig gegen die institutionellen Strukturen einer koordinierten Marktwirtschaft richtet (Höpner u. Schäfer 2007).

Mit anderen Worten, aus strukturellen Gründen (die nichts mit etwaigen ,neoliberalen“ Präferenzen der Richter zu tun haben) muss die richterliche Rechtssetzung einen asymmetrischen Einfluss auf die beiden Ländergruppen haben. Bei vollständiger Information

Verteilungskonsequenzen, die einer politischen Legitimierung bedürfen (Follesdal u. Hix, 2006; Hix 2008). 
hätten deshalb Mitgliedstaaten, die an der Erhaltung ihrer koordinierten Marktwirtschaft interessiert sind, einer Ermächtigung des Gerichts zur extensiven Interpretation des Vertrages nicht zustimmen können. Im Ergebnis können also weder das Dilemma-Argument noch das Incomplete-Contracting-Argument die politisch nicht kontrollierbare Ausweitung der richterlichen Rechtsetzung mit dem Verweis auf einen zwar virtuellen aber dennoch rationalen Konsens der Mitgliedstaaten legitimieren.

Faktisch stand diese Frage freilich auch nie auf der Tagesordnung einer europäischen Regierungskonferenz. Stattdessen expandierte das Richterrecht in Reaktion auf eine wachsende Unzufriedenheit mit dem Schneckentempo der politischen Gesetzgebung in der Gemeinschaft. Nach der Erweiterung der Gemeinschaft und in den wirtschaftlichen Krisen der siebziger Jahre war die Harmonisierung nationaler Handelsregeln kaum noch vorangekommen. Deshalb fand der Gerichtshof den Beifall vieler ,guter Europäer“, als er mit der Dassonville-Entscheidung ${ }^{12}$ und der Cassis-de-Dijon-Entscheidung ${ }^{13}$ begann, nicht-tarifäre Handelshindernisse als Verletzung der aus dem Vertrag abgeleiteten wirtschaftlichen Grundfreiheiten zu beseitigen.

Angesichts der scheinbar blockierten politischen Integration eröffneten diese Entscheidungen also einen zweiten, von den Mitgliedstaaten nicht mehr kontrollierbaren Weg zur „Integration durch (Richter-)Recht" ${ }^{\star 14}$ Gleichzeitig ging von diesen Entscheidungen ein neuer - ebenfalls asymmetrisch wirkender - Impuls zur politischen Integration aus. Die Cassis-Entscheidung hatte die Mitgliedstaaten mit der Gefahr konfrontiert, dass ihre eigenen Vorschriften durch das Prinzip der ,gegenseitigen Anerkennung“ ersetzt werden könnten - einer Gefahr, die, wann immer die Kommission es wollte, durch die Einleitung von Vertragsverletzungsverfahren aktualisiert werden konnte (S. Schmidt 2007; Nicolaidis u. Schmidt 2007). Im Vergleich zur unkontrollierbaren gerichtlichen Liberalisierung musste nun die Einigung auf harmonisierte Mindeststandards auch den Mitgliedstaaten mit koordinierter Marktwirtschaft als das kleinere Übel erscheinen. Im Ergebnis stimmten sie deshalb sowohl dem Programm zur Vollendung des Binnenmarktes als auch der Einheitlichen Europäischen Akte zu, die für die darauf gerichtete Gesetzgebung Entscheidungen mit qualifizierter Mehrheit ermöglichte. Und da die Cassis-Entscheidung die Verhandlungsmacht der Länder mit hohem Regulierungsgrad in vielen Bereichen ${ }^{15}$ nachhaltig geschwächt hatte, hatten auch die neuen Richtlinien eine stark liberalisierende und deregulierende Tendenz. Sie setzt sich fort, weil die Rechtsprechung auch weiterhin

12 Rs. C-8/74, 11.07.1974.

13 Rs. C-120/78, 20.20.1979.

14 „Integration through law“ ist der gemeinsame Titel der Serie von Bänden, die im Rahmen des berühmten „European legal integration project“ des Fachbereichs Rechtswissenschaft des European University Institute entstanden (Cappelletti et al. 1985a). Es sollte jedoch erwähnt werden, dass die Herausgeber der Serie die normativen und pragmatischen Ambivalenzen einer Divergenz zwischen rechtlicher und politischer Integration durchaus gesehen hatten (Cappelletti et al. 1985b).

15 Anderes gilt dort, wo das Gericht entgegen einer generellen Tendenz die Berechtigung handelsbeschränkender nationaler Regelungen anerkannt und damit die Position der Verteidiger eines hohen Regulierungsniveaus auch bei Verhandlungen über Harmonisierungsrichtlinien gestärkt hatte. 
die extensive Interpretation europarechtlich garantierter Individualrechte vorantreibt und damit die Verhandlungsbasis zum Nachteil nationalstaatlicher Regelungen verändert.

Die Ausweitung des europäischen Richterrechts ist ohne Mitwirkung der Mitgliedstaaten zustande gekommen, wurde aber von diesen im Wesentlichen ohne manifesten Widerstand hingenommen. Dafür gab es plausible Gründe. Nachdem die nationalen Gerichte die Prinzipien der Direktwirkung und der Suprematie einmal akzeptiert hatten, wurde das vom EuGH interpretierte Europarecht zum integralen Bestandteil der mitgliedstaatlichen Rechtsordnung. Der Widerstand hätte sich also gegen diese richten müssen und wäre einer Aufkündigung des prinzipiellen Rechtsgehorsams der Staatsgewalt gleichgekommen (Alter 2001). Das heißt aber auch, dass die bloße Hinnahme der EuGH-Entscheidungen durch die mitgliedstaatlichen Regierungen nicht als legitimierende Zustimmung zur Funktionsteilung zwischen richterlicher Rechtsetzung und politisch verantworteter Gesetzgebung gewertet werden kann (Möllers 2008).

Deshalb erscheint es auch naheliegend, die Rechtsprechung des EuGH mit der Legitimität des richterlichen Prüfungsrechts der nationalen Verfassungsgerichte zu vergleichen. Diese können sich in der Tat über die parlamentarische Gesetzgebung hinwegsetzen. Aber eben deshalb wird ja das richterliche Prüfungsrecht in Ländern mit einer starken demokratischen Tradition auch keineswegs als unproblematisch angesehen (Bickel 1962; Kramer 2004; Bellamy 2007; Wind et al. 2009). Jedenfalls aber können die für die nationale Verfassungsrechtsprechung entwickelten legitimierenden Argumente nicht unmittelbar zugunsten der Rechtsprechung des EuGH verwendet werden.

Die Rechtsprechung des nationalen Verfassungsgerichts ist in die nationale politische Kultur eingebettet, in der auf der Grundlage gemeinsamer Wertorientierungen eine normative und kognitive Verständigung über die Legitimität von Zielen und Mitteln der Politik als im Prinzip möglich unterstellt werden kann (March u. Olsen 1989). In solchen Diskursen spielen die Gerichte zwar eine wichtige Rolle, aber sie sind keineswegs die einzig legitimierten Interpreten der gemeinsamen Wertorientierung. Sie müssen davon ausgehen, dass die Verpflichtung auf die gemeinsamen Werte von allen Organen des Gemeinwesens geteilt wird und dass alle politisch Verantwortlichen geschworen haben, die verfassungsmäßige Ordnung zu wahren. Sie werden deshalb parlamentarische Gesetze im Geiste richterlicher Selbstbeschränkung und mit der Vermutung ihrer Verfassungsmäßigkeit prüfen. Und wenn sie trotzdem gegen aktuelle Mehrheiten intervenieren müssen, so beruht die Überzeugungskraft des Urteils letztlich auf seiner Resonanz mit dem grundlegenden Wertkonsens des Gemeinwesens - „the sober second thought of the community“ (Bickel 1962, S. 26; Fisher 1988; Höreth 2008).

Im Verhältnis zwischen dem EuGH und den Mitgliedstaaten fehlen die Voraussetzungen, die das richterliche Prüfungsrecht zugleich begrenzen und legitimieren. Das mag im Verhältnis des Gerichts zur Kommission und zum Europäischen Parlament anders sein. Aber von einer Einbettung des Gerichts in gemeinsame Wertorientierungen und Situationsdeutungen mit den Regierungen, den Parlamenten und den öffentlichen Diskursen in den siebenundzwanzig außerordentlich heterogenen Mitgliedstaaten der Union könnte in der Tat nicht die Rede sein. Deshalb gibt es weder die Maximen der richterlichen Selbstbeschränkung noch die Vermutung der Vertragskonformität, wenn der Gerichtshof Gesetze der Mitgliedstaaten zu prüfen hat. Stattdessen erscheint die europäische Integration aus Sicht des Gerichtshofes als eine Mission, die gegen die Trägheit oder Widerspenstigkeit 
von Mitgliedstaaten erfüllt werden muss, und das europäische Recht ist nicht der Ausdruck einer gemeinsamen Wertorientierung, sondern ein Instrument zur Disziplinierung und Transformation nationaler Politiken, Institutionen und Praktiken.

Wohin hat uns diese Diskussion nun geführt? Selbstverständlich steht die formale Legalität der Rechtsprechung des Gerichtshofes außer Frage. Art. 220 EG enthält ein klares Mandat zur Anwendung und Auslegung des Europarechts. Juristen mögen über manche seiner Interpretationen streiten, aber sie werden diese nicht als ultra vires qualifizieren. ${ }^{16}$ Angesichts von widersprüchlichen Formelkompromissen, unbestimmten Generalklauseln und gewollter Mehrdeutigkeit im europäischen Primär- und Sekundärrecht wäre das Gericht ohnehin oft überfordert, wenn es den „ursprünglichen Willen“ der „Herren der Verträge“ oder des multiplen europäischen „Gesetzgebers“ ermitteln wollte. Aber wie die Europäer durch bittere Erfahrungen lernen mussten, impliziert die formale Legalität nicht unbedingt auch die Legitimität des anzuwendenden Rechts (Joerges u. Ghaleigh 2003). Sie reicht gewiss, um in einem im Grundsatz als legitim betrachteten Gemeinwesen die Hinnahme der alltäglichen Gebote und Verbote der Staatsgewalt zu sichern. Aber wenn politisch hochsensible Interessen und normative Präferenzen verletzt werden, dann müssen zur formalen Legalität positiv legitimierende Argumente hinzukommen, um die Routinen der freiwilligen Folgebereitschaft zu stabilisieren.

Im Verhältnis zwischen den Mitgliedstaaten und der EU haben die römisch-rechtlichen Maximen pacta sunt servanda und volenti non fit iniuria zweifellos erhebliches Gewicht. Ihre Regierungen oder deren Vorgänger haben die heutigen Institutionen der EU mitgestaltet, und die Regierungen der neueren Mitgliedstaaten haben mit dem Beitritt die vorhandenen Institutionen und den akkumulierten acquis des Gemeinschaftsrechts wissentlich akzeptiert. Aber diese Verpflichtungen werden durch die dritte römische Maxime „ultra posse nemo obligatur“ beschränkt. Wie ich schon gesagt habe, stößt die Fähigkeit der Mitgliedstaaten, das Gemeinschaftsrecht zu implementieren, an ihre Grenzen, wenn dessen Befolgung die Grundlagen ihrer Legitimationsbeziehung zum eigenen Staatsvolk gefährden würde. In den folgenden Abschnitten will ich zuerst die allgemeinen Bedingungen dieser Beziehung erörtern und mich dann einer Reihe neuerer Entscheidungen zuwenden, bei denen die Rechtsprechung des EuGH an die Grenzen der legitimen Folgebereitschaft der Mitgliedstaaten zu stoßen scheint.

\section{Die Notwendigkeit einer Rechtfertigung}

Da das Recht der Union von ihren Mitgliedstaaten umgesetzt und vollzogen werden muss, ist es die Legitimität des Mitgliedstaates, die die Folgebereitschaft der Bürger zu gewährleisten hat. Wie oben ausgeführt, ruht diese Legitimität sowohl auf ,liberalen“ als auch auf ,republikanischen“ Grundlagen. Im Großen und Ganzen ist es jedoch unwahrschein-

16 Kennzeichen der Jurisprudenz des EuGH ist die Dominanz der teleologischen Auslegungsmethode, mit der die maximale Wirksamkeit des Vertrages gesichert werden soll (,effet util“). Aber diese problematische Tendenz zur Vernachlässigung der historischen und philologischen Interpretation scheint auch in der nationalen Jurisprudenz um sich zu greifen (Lübbe-Wolf 2007). 
lich, dass das durch Richterrecht geschaffene Gemeinschaftsrecht die spezifisch liberalen Prinzipien der nationalen Verfassungen verletzt. ${ }^{17}$ Dagegen könnte die „republikanische“ Legitimität der Mitgliedstaaten in der Tat auf dem Spiel stehen.

Republikanische Demokratie erfordert ja nicht nur die formale Existenz regelmäßiger, allgemeiner und gleicher Wahlen und repräsentativer Parlamente, sie setzt auch voraus, dass die Verantwortlichkeit der Regierenden gegenüber der Wählerschaft sich auf den Inhalt staatlicher Politik auswirken kann. Dieses (input-orientierte) Erfordernis impliziert mindestens, dass die Regierenden die Interessen und Präferenzen ihrer Bürger berücksichtigen sollen, und sie impliziert auch, dass die Abwahl der Regierung einen Einfluss auf Politiken haben kann, die von einer Mehrheit der Wähler nachdrücklich abgelehnt werden. Aber zugleich haben Regierungen auch eine „republikanische“ (output-orientierte) Pflicht, die Staatsgewalt zur Förderung des Gemeinwohls einzusetzen. In den normativen Traditionen konstitutioneller Demokratien sind beide Pflichten von grundlegender und gleichrangiger Bedeutung. Aber ihre Implikationen können sich widersprechen, wenn auf das öffentliche Interesse gerichtete Programme unpopulär sind, populäre Programme aber das öffentliche Interesse gefährden würden. Im Falle einer solchen Pflichtenkollision maß die normative politische Theorie von Aristoteles bis Edmund Burke der Verpflichtung auf das öffentlichen Interesse Priorität bei. Dagegen waren sogar Demokratietheoretiker, die die paternalistischen oder technokratischen Implikationen output-orientierter Legitimationsargumente kritisieren (Greven 2000; Bartolini 2005; Hix 2008), kaum je bereit, den radikalen Populismus als eine normativ akzeptable Position zu verteidigen (Mény u. Surel 2002).

Statt dessen richten moderne Varianten einer input-orientierten Demokratietheorie die Aufmerksamkeit auf die kommunikative Interaktion zwischen Regierenden und Regierten. Verantwortliche Regierungen sind zwar dem Gemeinwohl verpflichtet. Aber dessen substantielles Verständnis und die Maßnahmen, die seiner Verwirklichung dienen, sind keine Fragen des Expertenwissens, sondern sie sollen aus der diskursiven Interaktion im öffentlichen Raum des Gemeinwesens hervorgehen (Habermas 1962, 1992, 2008; Dryzek 2000; Greven 2000; de Vreese u. Schmitt 2007). Mit Blick auf die Möglichkeiten der politischen Praxis richtet Vivien Schmidt $(2004,2006)$ die Aufmerksamkeit auf die Rolle politikorientierter „kommunikativer Diskurse“, in denen die Regierenden eine unpopuläre Politik, die sie für notwendig und normativ vertretbar halten, in der öffentlichen Auseinandersetzung mit ihren Kritikern erklären und rechtfertigen müssen. Je wichtiger die Interessen sind, die verletzt werden können, und je weiter die beabsichtigte Politik von intensiven normativen Überzeugungen der Wähler abweicht, um so dringlicher sind Erklärungen und rechtfertigende Argumente, die zeigen könnten, dass und wie die umstrittenen Vorhaben unter den gegebenen Bedingungen dem Gemeinwohl dienen und mit den Werten des Gemeinwesens vereinbar bleiben.

17 Der Schutz der Menschenrechte war noch ungewiss, als das deutsche Bundesverfassungsgericht in seinen „Solange-Beschlüssen“ die Möglichkeit erwog, die Verfassungsmäßigkeit von EURecht zu prüfen - BVerfG 37, 271/29.05.1974), BVerfG 73, 339 (22.10.1986). Inzwischen hat der EuGH reagiert, und das deutsche Gericht hat den Grundrechtsvorbehalt aufgegeben (Weiler u. Lockhart 1995). 
Wenn die Wähler in solchen kommunikativen Diskursen überzeugt werden können, bleibt die input-orientierte Legitimität der Politik gewahrt. Wenn dies nicht gelingt, sind demokratische Regierungen potenziell gefährdet. Zwar sind unter normalen Umständen die allgemeinen Wahlen gewiss kein zielgenauer und sensibler Mechanismus zur Sanktionierung einzelner politischer Entscheidungen. Die Wähler haben ja nur eine Stimme, mit der sie ihre Zustimmung oder ihren Unmut über eine Vielzahl politischer Entscheidungen, über allfällige Skandale und die persönlichen Meriten führender Kandidaten zum Ausdruck bringen können. Und selbst wenn sich der Protest gestern auf eine bestimmte Frage konzentrierte, kann er bis zur nächsten Wahl aus der öffentlichen Wahrnehmung verschwunden sein. ${ }^{18}$ Aber wenn eine Politik die vitalen Interessen oder intensiven normativen Überzeugungen der Wählerschaft verletzt, dann kann eine Regierung, die an ihrem Kurs festhält, ohne überzeugen zu können, in der Tat bei der nächsten Wahl scheitern. ${ }^{19}$ Wenn das geschieht, dann wird zwar nicht die fragliche Politik legitimiert, aber es wird die institutionelle Legitimität eines politischen Systems mit einer verantwortlichen und demokratisch rechenschaftspflichtigen Regierung bestätigt.

Das Gegenteil ist jedoch der Fall, wenn eine Politik, die den vitalen Interessen und normativen Überzeugungen der nationalen Wählerschaft zuwiderläuft, nicht unter Bezug auf die Werte des Gemeinwesens in kommunikativen Diskursen erklärt und gerechtfertigt werden kann. Wird sie dennoch durchgesetzt, so kann dies die Legitimität konstitutioneller Demokratien untergraben und am Ende womöglich zerstören. Dieses Risiko ist zu bedenken, wenn nationale Regierungen europäisches Recht implementieren müssen, das ohne Mitwirkung politisch verantwortlicher Akteure durch richterliche Rechtssetzung geschaffen worden ist. Das soll nicht heißen, dass das von Richtern gesetzte europäische Recht, das politisch wichtigen Interessen und tief verwurzelten normativen Überzeugungen in den Mitgliedstaaten widerspricht, nie als notwendig und angemessen gerechtfertigt werden könnte. Aber die Rechtfertigung erscheint hier schwieriger als im Falle der europäischen Gesetzgebung, an der die Regierungen beteiligt sind und für die sie deshalb gute Gründe zu nennen in der Lage sein müssten.

Grundsätzlich könnte das Richterrecht mit zwei Argumenten verteidigt werden. Das erste würde an das ,aufgeklärte“ nationale Eigeninteresse appellieren. Es würde zu zeigen versuchen, dass das Land, wenn man alle Umstände berücksichtigt, vom Vollzug der fraglichen europäischen Norm größere Vorteile zu erwarten hat, als vom Verzicht auf eine

18 In realen Demokratien kann die politische Effektivität des Wahlmechanismus trotzdem recht hoch sein: In Deutschland wird die Bundesregierung während ihrer vierjährigen Amtszeit in 16 Landtagswahlen getestet; in allen Konkurrenzdemokratien werden Oppositionsparteien ihr Bestes versuchen, um vor der nächsten Wahl das Gedächtnis der Wähler aufzufrischen, und in jedem Fall können Regierungen nicht schon vorher wissen, welche Frage schließlich für welche Wähler entscheidend sein wird. Falls sie überhaupt dazu in der Lage sind, werden sie deshalb nach der „Regel der antizipierenden Reaktion“ versuchen, auf alle erkennbaren Beschwerden der Wähler zu reagieren (Scharpf 1997, S. 183-188).

19 Dies war etwa der Fall, als die niederländische Regierung Anfang der neunziger Jahre die Berufsunfähigkeitsrenten reformierte (Hemerijck et al. 2000, S. 220-224), und das Gleiche geschah in Deutschland, als die Schröder-Regierung trotz Massenprotesten und rasch abnehmender öffentlicher Unterstützung an ihren „Agenda-2010“-Reformen festhielt (Egle u. Zohlhöfer 2007). 
europäische Regelung. Im Wesentlichen geht es dabei also um Argumente, die auch eine Einigung bei politischen Verhandlungen ermöglichen würden, sofern die zugrundeliegende Interessenkonstellation den spieltheoretischen Modellen des „Versicherungsspiels“, des „Kampfes der Geschlechter“ oder des (symmetrischen) „Gefangenendilemmas“ ähneln (Scharpf 1997, Kap. 6). Aber wie verhält es sich bei Nullsummenspielen oder bei asymmetrischen Konstellationen, in denen eine europäische Regelung einem Teil der Mitgliedstaaten einseitige Opfer abverlangt?

Wenn die europäische Norm gar nicht im Sinne des aufgeklärten Eigeninteresses des Mitgliedstaates gerechtfertigt werden kann, gäbe es aus analytischer Sicht noch die Möglichkeit, einseitige nationale Opfer unter Berufung auf das kollektive Eigeninteresse der Union zu rechtfertigen. Diese Rechtfertigung würde jedoch je nach Größe des geforderten Opfers eine kollektive europäische Identität voraussetzen, die stark genug wäre, um das nationale Eigeninteresse zu delegitimieren. Aber leider ist dies eine Voraussetzung, die auch enthusiastische „Europäer“ gegenwärtig nicht als erfüllt ansehen (Pollak 2008).

Das heißt allerdings nicht, dass sich asymmetrische nationale Opfer niemals in nationalen Diskursen rechtfertigen ließen. Die überzeugendste Rechtfertigung liegt gewiss in den Errungenschaften der europäischen Integration selbst. Die Union hat bisher nicht die dem Muster erfolgreicher Bundesstaaten nachgebildeten „Vereinigten Staaten von Europa" hervorgebracht (Nicolaidis u. Howse 2003). Und vielleicht wird und sollte sie dieses Ziel auch nie erreichen. Immerhin aber hat die europäische Integration den Frieden zwischen europäischen Nationen erhalten, die sich über Jahrhunderte in Bruderkriegen zerfleischt hatten. Und sie konnte den Übergang zur Demokratie und die Achtung der Menschenrechte auf einem Kontinent gewährleisten, der die verbrecherischsten Regime der Menschheitsgeschichte hervorgebracht hat. Diese Ergebnisse hätten nicht durch die rücksichtslose Durchsetzung nationaler Eigeninteressen erzielt werden können. Die Zugehörigkeit zur Union setzt Mitgliedstaaten voraus, deren Institutionen und deren Politik mit den Grunderfordernissen europäischer Kommunalität vereinbar sind. Das heißt, dass nationale Präferenzen durch die normative Verpflichtung zur „Einbeziehung des Anderen“ (Habermas 1996) und durch das „Prinzip der Verfassungstoleranz “ (Weiler 1999b; 2003) so modifiziert werden, dass die Durchsetzung eigener Optionen zum Schaden der Gemeinschaft nicht mehr angemessen erscheint. Die Bewahrung der gemeinschaftlichen Errungenschaften kann Beschränkungen der nationalen Autonomie auch dann rechtfertigen, wenn dadurch politisch wichtige Interessen und Präferenzen in den Mitgliedstaaten verletzt werden. Und europäische Regeln, die die Voraussetzungen der Kommunalität schützen, können mit guten Gründen verteidigt werden, gleich ob sie nun im politischen oder unpolitischen Modus der europäischen Politik beschlossen wurden. Und wo dies der Fall ist, da können und sollten sie auch von den Regierungen der Mitgliedstaaten selbst gegen starken innerstaatlichen Widerstand verteidigt werden.

\section{Der Gerichtshof stößt an die Grenzen der Legitimierbarkeit}

Abstrakt gesehen erscheinen die Legitimationsargumente zugunsten der republikanischen Selbstbestimmung in den Mitgliedstaaten und zugunsten der normativen Anforderungen der europäischen Kommunalität gleichermaßen gewichtig. Deshalb muss im Konfliktfall 
das relative Gewicht der im konkreten Fall auf dem Spiel stehenden Wertverletzungen gegeneinander abgewogen werden. Je größer die politische und normative Bedeutung der nationalen Institutionen und Politikstrukturen, die angegriffen werden, desto gewichtiger müsste die behauptete Vertragsverletzung sein, wenn sie ein Verbot rechtfertigen soll. Freilich hatte die Rechtsprechung mehrere Jahrzehnte lang keinen Anlass, klare Kriterien für diese normative Abwägung zu entwickeln. Europarechtliche Fragen fanden kaum je das Interesse der Öffentlichkeit in den Mitgliedsstaaten: Und das Gericht selbst scheint darauf bedacht gewesen zu sein, die rechtlichen Grundsätze in Entscheidungen zu entwickeln, bei denen es in der Sache eher um politisch unspektakuläre, um nicht zu sagen triviale Streitgegenstände ging. In der Cassis-Entscheidung beispielsweise postulierte das Gericht zum ersten Mal die den Geltungsbereich mitgliedstaatlichen Rechts radikal beschränkenden Grundsätze der wechselseitigen Anerkennung und des Herkunftslandsprinzips. Aber im konkreten Fall ging es um die Zulassung eines französischen Likörs, dessen Alkoholgehalt nach deutscher Vorschrift zu niedrig war - ein Thema, über das sich gewiss niemand politisch echauffieren konnte. Frühere Warnungen vor den Folgen der Rechtsprechung des EuGH für das Überleben der nationalen Sozialsysteme (vgl. z.B. Scharpf 1999) ließen sich deshalb leicht als unrealistisch verwerfen (Moravcsik u. Sangiovanni 2003). Heute aber, wo die Grundsätze fest in der ständigen Rechtsprechung verankert und von den nationalen Gerichten anerkannt sind, scheinen der Europäische Gerichtshof und die Kommission entschlossen zu sein, auch ernsthaften politischen Konflikten nicht mehr auszuweichen. Ich möchte deshalb kurz auf einige neuere Entscheidungen eingehen, die diese stärker autonomiebeschränkende und potentiell legitimationsgefährdende Tendenz der Rechtsprechung verdeutlichen.

Der erste Fall hat nichts mit den neoliberalen Präferenzen zu tun, die dem Europäischen Gerichtshof und der Kommission oft nachgesagt werden. Österreich, wo das Hochschulstudium unentgeltlich und allen Absolventen eines Gymnasiums zugänglich ist, musste erleben, wie seine medizinischen Fakultäten von Studienbewerbern aus Deutschland überflutet wurden, deren Schulabschlüsse nicht gut genug für eine Zulassung nach den deutschen Numerus-clausus-Regeln waren. Die Regierung reagierte darauf mit einer Vorschrift, derzufolge ausländische Studienbewerber nachweisen mussten, dass sie auch für ein Medizinstudium in ihrem jeweiligen Heimatland qualifiziert waren. Die Kommission leitete daraufhin ein Vertragsverletzungsverfahren ein, und der EuGH befand, dass die österreichische Vorschrift das Freizügigkeitsrecht der Studenten verletze und gegen den Grundsatz der Nichtdiskriminierung gemäß Art. 12 EG verstoße. ${ }^{20}$ Diese Entscheidung führte unmittelbar dazu, dass an einigen medizinischen Fakultäten in Österreich mehr als 60 Prozent der Studienbewerber aus Deutschland kamen.

In einer zweiten Serie neuerer Entscheidungen ging es dann in der Tat um den Vorrang der Wirtschaftsfreiheiten vor sozialen Rechten, die in den Verfassungen der Mitgliedstaaten geschützt werden. Im Viking-Fall ${ }^{21}$ hatte eine finnische Reederei, die von Helsinki aus operierte, die Umflaggung eines Fährschiffes von Finnland auf Estland beschlossen. Die finnische Gewerkschaft drohte mit Streik und die Reederei beantragten dessen Verbot. Der Fall wurde zur Vorabentscheidung dem EuGH vorgelegt, der den Streik als Beein-

20 Rs. C-147/03, 07.07.2005.

21 Rs. C-438/05, 11.12.2007. 
trächtigung der Niederlassungsfreiheit des Unternehmens bewertete. Im Laval-Fall ${ }^{22}$ weigerte sich ein lettisches Unternehmen, das eine Schule in Schweden baute, über Löhne zu verhandeln, die dem Mindestlohniveau der schwedischen Tarifverträge entsprachen. Der EuGH bewertete die Aktion der schwedischen Gewerkschaft als einen Verstoß gegen die Dienstleistungsfreiheit des Unternehmens, der nicht durch eine enge Auslegung der Richtlinie über die Entsendung von Arbeitnehmern gedeckt sei. ${ }^{23}$

Waren der Viking- und der Laval-Fall gegen das verfassungsmäßige Recht der finnischen und schwedischen Gewerkschaften gerichtet, Kampfmaßnahmen zur Wahrung kollektiver Interessen zu ergreifen, so stehen die Fälle Rüffert ${ }^{24}$ und Luxemburg ${ }^{25}$ für die Durchsetzung des Vorrangs der Dienstleistungsfreiheit vor dem nationalen Tarifrecht. Im Fall Rüffert ging es um das niedersächsische Tariftreuegesetz, demzufolge bei öffentlichen Aufträgen Löhne entsprechend dem regionalen Tarifvertrag zu zahlen waren. Luxemburg dagegen hatte die europäische Entsenderichtlinie in einem Gesetz konkretisiert, das von allen Unternehmen die Einhaltung des im Land geltenden Arbeitsrechts, einschließlich der automatischen Lohnanpassung an die Inflationsrate verlangte. In beiden Fällen interpretierte der Gerichtshof die Richtlinie als Maximal- und nicht als Mindeststandard - mit der Konsequenz, dass darüber hinausgehende gesetzliche Regelungen als Beschränkung der Dienstleistungsfreiheit gewertet wurden.

In anderen Entscheidungen werden die Niederlassungsfreiheit und Kapitalverkehrsfreiheit in einer Weise interpretiert, die die Möglichkeiten der Mitgliedstaaten zur Gestaltung der in der heimischen Wirtschaft geltenden Unternehmensverfassung gravierend beschränken (Höpner u. Schäfer 2007). ${ }^{26}$ Zugleich hat die Rechtsprechung zur Kapitalverkehrsfreiheit die Möglichkeiten einer Besteuerung des mobilen Kapitals erheblich eingeschränkt (Ganghof u. Genschel 2008b; Genschel et al. 2008). Die hier wirksame Entscheidungslogik begünstigt jedoch nicht nur wirtschaftliche Interessen. Auch in der langen Reihe neuerer Entscheidungen, die EU-Bürgern Zugang zu den öffentlichen Einrichtungen und Sozialleistungen in anderen Mitgliedstaaten verschaffen (Ferrera 2005; Martinsen 2005, 2009; Martinsen u. Vrangbaek 2008; Wollenschläger 2007), gibt der Gerichtshof den Individualrechten auf Freizügigkeit und Nichtdiskriminierung Vorrang vor dem Prinzip der Reziprozität zwischen Rechten und Beiträgen im solidarischen Gemeinwesen.

\section{Die liberale Unterminierung der republikanischen Legitimität}

In diesen und in anderen Entscheidungen hat der Europäische Gerichtshof offensichtlich gegen wichtige und politisch höchst bedeutsame Gesetze, Institutionen und Praktiken der einzelnen Mitgliedstaaten interveniert. Doch warum sollte es unmöglich sein, diese Inter-

22 Rs. C-341/05, 18.12.2007.

23 Richtlinie 96/71 EG.

24 Rs. C-346/06, 03.04.2008.

25 Rs. C-319/06, 19.06.2008.

26 Vgl. z.B. Rs. C-219/97, 09.03.1999 (Centros); Rs. C-112/05, 23.10.2007 (Volkswagen). 
ventionen in nationalen kommunikativen Diskursen zu rechtfertigen? Die Wurzel des Problems liegt in der grundlegenden Asymmetrie der juristischen Instrumente, mit denen der Gerichtshof den Ausgleich zwischen den legitimen Autonomie-Belangen der Mitgliedstaaten und den legitimen Erfordernissen der europäischen Gemeinschaft definiert. ${ }^{27}$ Sie hat ihren Ursprung schon im Falle Van Gend en Loos (1963), der ersten Entscheidung, die die unmittelbare Anwendbarkeit des Gemeinschaftsrechts postulierte. ${ }^{28} \mathrm{Um}$ diese Doktrin zu etablieren, musste der Gerichtshof die vertragliche Pflicht, bestehende Zolltarife nicht mehr zu erhöhen, als das einklagbare Recht eines Unternehmens gegen den Staat interpretieren. In Verbindung mit dem fast gleichzeitig postulierten Vorrang des Gemeinschaftsrechts ${ }^{29}$ ermöglichte es diese Konstruktion dem Gerichtshof, immer weitere subjektive Rechte gegen die Mitgliedstaaten anzuerkennen und deren Anwendungsbereiche auszudehnen und so das jeweils von der nationalen Politik geschaffene Gleichgewicht zwischen den Rechten und Pflichten der Staatsangehörigen in die liberale Richtung zu verschieben.

Da die Verpflichtungen im ursprünglichen EWG-Vertrag in erster Linie die wirtschaftliche Integration herbeiführen sollten, erklärt deren Transformation in ,wirtschaftliche Grundfreiheiten“ die stark „marktliberalen“ Auswirkungen der EuGH-Rechtsprechung. Jedoch ist der Gerichtshof da, wo ihm das europäische Recht Handhaben dafür bot, auch gegen die nationale Beschränkung nicht-wirtschaftlicher Individualrechte vorgegangen. Dies gilt seit langem für Entscheidungen zur Durchsetzung und Erweiterung der Gleichheit von Männern und Frauen am Arbeitsplatz entsprechend Art. 141 EG (Cichowski 2004). Es gilt heute auch für die nicht-arbeitsmarktbezogenen Rechte der EU-Bürger auf Freizügigkeit und Nichtdiskriminierung aus Gründen der Nationalität (Wollenschläger 2007). Diese Rechtsprechung wurde von manchen als fundamentale Umkehrung der marktliberalen Tendenz des EuGH gepriesen (Caporaso 2006; Caporaso u. Tarrow 2008) - obwohl es sich doch nur um die Erstreckung der negativen Integration und Liberalisierung auf neue Gebiete handelt.

In dem vom EuGH geschaffenen Rahmen werden die europäischen Belange, die möglicherweise eine Beschränkung der nationalen Selbstbestimmung rechtfertigen können, als subjektive Rechte von Personen und Firmen definiert, statt als substantielle Erfordernisse, von denen die Funktionsfähigkeit der europäischen Staatengemeinschaft - oder auch des Binnenmarktes - abhängt. In Verbindung mit dem Vorrang des Gemeinschaftsrechts hat dieser Ansatz die Konsequenz, dass das hierarchische Verhältnis zwischen dem europäischen und dem nationalen Recht zugleich ein hierarchisches Verhältnis zwischen liberalen und republikanischen Verfassungsprinzipien erzeugt. ${ }^{30}$ Die subjektiven Rechte, die sich von dieser Auslegung des europäischen Rechts herleiten, sind prinzipiell in der

27 Wie Joe Weiler (1999a) in einem anderen Zusammenhang erläutert hat, geht es hier - jedenfalls zunächst - nicht um die Kompetenz-Kompetenz im europäischen Mehrebenensystem, sondern um die politischen Konsequenzen, die sich aus der asymmetrischen Logik der Rechtsprechung des EuGH ergeben.

28 Rs. C-26/62, 05.02.1963.

29 Rs. C-6/64, 15.07 .1964 (Costa/ENEL).

30 Richard Münch (2008b, S. 540) hat die durch die Jurisdiktion des EuGH geschaffene Rechtsordnung als „,für konkurrierende ökonomische Akteure gemacht“ gekennzeichnet. „Sie ist mehr 
Lage, alle entgegenstehenden nationalen Zielsetzungen und demokratisch legitimierten Institutionen zu übertrumpfen.

Angesichts der Unmöglichkeit politischer Korrekturen kann die Rechtsprechung bei der expansiven Interpretation europäischer Rechte ganz der immanenten Logik juristischer Argumente folgen. Auf dem Gebiet des Freihandels zum Beispiel untersagt der Vertrag quantitative Beschränkungen der Ein- und Ausfuhr von Waren und „Maßnahmen, die den gleichen Effekt haben“ (Art. 28 EG). Ursprünglich wurde dies als Verbot der diskriminierenden Behandlung von Importen verstanden. Anfang der 1970-er Jahre wurde diese Auffassung jedoch von der radikaleren Dassonville-Formel verdrängt, wonach ,jede Handelsregelung der Mitgliedstaaten, die geeignet ist, den innergemeinschaftlichen Handel unmittelbar oder mittelbar, tatsächlich oder potentiell zu behindern, [...] als Maßnahme mit gleicher Wirkung wie eine mengenmäßige Beschränkung anzusehen [ist]. " ${ }^{\prime 31}$ Es bedarf also keiner beabsichtigten oder faktischen Diskriminierung mehr, und es genügt schon eine hypothetische Behinderung des Freihandels, der freien Kapitalbewegung, des freien Dienstleistungsverkehrs oder der Niederlassungsfreiheit, um eine nationale Bestimmung außer Kraft zu setzen.

Freilich wird die Dassonville-Formel nicht als unbedingtes Verbot gehandhabt. Der Vertrag selbst formuliert in Verbindung mit den einzelnen Liberalisierungstatbeständen jeweils auch bestimmte Rechtfertigungsgründe für mitgliedstaatliche Beschränkungen des freien Verkehrs. Und in Cassis-de-Dijon hat das Gericht zu den im Vertrag genannten Gründen noch eine Generalklausel hinzugefügt, mit der „zwingende Erfordernisse des öffentlichen Interesses“ vom Beschränkungsverbot ausgenommen werden können (Haltern 2007, S. 742-755). Aber auch wenn sich damit die Möglichkeit einer Abwägung zwischen europäischen Rechten und nationalen Belangen eröffnet, wird diese zum Nachteil nationaler Lösungen doch wieder in asymmetrischer Weise verwirklicht. Der Effekt zeigt sich in drei Dimensionen:

Erstens entscheidet das Gericht darüber, welche nationalen Politikziele überhaupt als „zwingende Erfordernisse des öffentlichen Interesses“ in Frage kommen. Fiskalische Belange beispielsweise werden grundsätzlich nicht berücksichtigt. So wurden etwa in der oben erwähnten Österreich-Entscheidung die Auswirkungen des massenhaften Zustroms deutscher Studenten auf die Hochschulhaushalte völlig ignoriert. Das Gleiche gilt für Entscheidungen, die im Interesse der Freizügigkeit die nationalen Sozialleistungssysteme für Migranten öffnen, ${ }^{32}$ oder die im Interesse der Dienstleistungsfreiheit die nationalen Gesundheits- und Sozialversicherungssysteme verpflichten, für im Ausland in Anspruch genommene Leistungen aufzukommen (Martinsen 2005, 2009). ${ }^{33}$ Ebenso bleiben fiska-

für den Marktbürger geeignet als für den politischen Bürger des Republikanismus oder für den sozialen Bürger der Sozialstaaten im sozialdemokratischen oder konservativen Sinne.“

31 Rs. C-8/74, 111.07.1974.

32 Vgl. z.B Rs. C-10/90, 07.03.1991 (Masgio); Rs. C-245/94; Rs. C-312/94, 10.10.1996 (Hoever u. Zachow); Rs. C-131/96, 25.06.1997 (Romero); Rs. C-160/96, 05.03.1998 (Molenaar); Rs. 85/96, 12.05 .1998 (Sala).

33 Vgl. z.B. Rs. C-120/95, 28.04.1998 (Decker); Rs. C-158/96, 28.04 .1998 (Kohll); Rs. C-157/99, 12.07.2001 (Geraets-Smits u. Peerbooms); Rs. C-385/99, 13.05.2003 (Müller-Fauré u. Van Riet). 
lische Interessen außer Betracht, wenn nationale Regeln gegen Steuerflucht als Behinderungen des freien Kapitalverkehrs angegriffen werden (Ganghof u. Genschel 2008b).

Indem der Gerichtshof die fiskalischen Implikationen der Inanspruchnahme europäischer Freiheiten für irrelevant erklärt, zerstört er den Zusammenhang zwischen den Rechten und Pflichten der Mitgliedschaft in einem Gemeinwesen, und er ignoriert die legitimatorische Bedeutung, die dem parlamentarischen Budget-Monopol in allen konstitutionellen Demokratien zukommt (Ganghof u. Genschel 2008a). Aus republikanischer Perspektive hätten die deutschen Studenten gute Gründe, um gegen die unzureichende Hochschulfinanzierung im deutschen Föderalismus zu protestieren - aber man käme gewiss nicht auf die Idee, ihnen statt dessen einen Anspruch gegenüber den österreichischen Steuerzahlern zuzuerkennen. Das Gleiche gilt für andere steuerfinanzierte Dienstleistungen, für soziale Transferleistungen und auch für Systeme einer gesetzlichen Krankenversicherung, bei denen mit der Gesamtsumme der Beiträge ein ausreichendes Gesamtangebot finanziert werden muss. ${ }^{34}$ Ebenso wenig lässt es sich aus republikanischer Sicht rechtfertigen, wenn Unternehmen und Individuen in die Lage versetzt werden, die öffentliche Infrastruktur und die öffentlichen Dienstleistungen eines Landes zu nutzen, ohne auch Beiträge zu dem Steueraufkommen zu leisten, mit dem diese Vorteile finanziert werden.

Der Gerichtshof wirkt vielleicht generös, wenn er die reziproke Verbindung von Ansprüchen und Beiträgen durch europäische Grundrechte und das Diskriminierungsverbot außer Kraft setzt. Aber seine Generosität ignoriert den Klubgut-Charakter der Leistungen des solidarischen Nationalstaates. Im theoretischen Modell steht die Existenz eines Klubs auf dem Spiel, wenn den Beitragszahlern der Austritt und den Nichtzahlern der Zutritt zu sehr erleichtert wird. Wenn deshalb die Politik in den Mitgliedstaaten auf die EuGH-Rechtsprechung modellgerecht reagieren sollte, so wäre das erwartbare Ergebnis gerade nicht die diskriminierungsfreie und europaweite Generosität öffentlicher Leistungen. Wahrscheinlicher wären auf der einen Seite (europaweit operierende) private Versicherungen, private Schulen und Hochschulen und bewachte Ghettos für die Besserverdienenden und auf der anderen Seite die Erosion öffentlicher Sozialleistungen, öffentlicher Dienste und öffentlicher Infrastruktur für diejenigen (einschließlich der nicht länger diskriminierten Migranten), die für private Lösungen nicht zahlen können.

Die zweite Asymmetrie betrifft die „Abwägung“ in den Politikfeldern, in denen die nationale Regelung sich auf einen vom Gericht grundsätzlich anerkannten Rechtfertigungsgrund berufen kann. Dabei geht es niemals darum, wie gravierend die im konkreten Fall zu erwartende Beschränkung der Grundfreiheiten sich auf die europäischen Belange auswirken könnte. Ganz gleich, wie trivial die Belastung der Freiheitsrechte im konkreten Fall sein mag, die Abwägung findet immer nur auf der Seite der vom Mitgliedstaat vorgetragenen Rechtfertigungsargumente statt, die in jedem Falle einer rigorosen materiel-

34 Damit will ich nicht bestreiten, dass die „Einbeziehung der Anderen“ es gebieten kann, in vielen Fällen Leistungen an Gebietsfremde auch ohne Beitragsbeteiligung zu gewähren. Wenn diese Verpflichtung fiskalischen Belangen systematisch untergeordnet wird, kann dies tatsächlich durch gerichtliche Intervention im Einzelfall korrigiert werden. Aber die Frage nach einem angemessenen Ausgleich stellt sich erst gar nicht, wenn fiskalische Erwägungen von vornherein als irrelevant angesehen werden. 
len und prozeduralen Verhältnismäßigkeitsprüfung unterworfen werden. ${ }^{35}$ Hier genügt es nicht, dass der nationale Gesetzgeber einen bestimmten Zweck für erstrebenswert oder ein bestimmtes Rechtsgut für schutzwürdig erklärt hat: nur das Gericht selbst kann entscheiden, ob es dabei aus europäischer Perspektive um ein ,zwingendes Erfordernis des öffentlichen Interesses“" geht. Aber damit nicht genug. Nun muss die nationale Regierung (erstens) das Gericht davon überzeugen, dass die vom Gesetzgeber gewählte Maßnahme auch tatsächlich geeignet ist, das akzeptierte Ziel zu erreichen, und sie muss (zweitens) auch noch beweisen, dass der angestrebte Effekt nicht auch durch andere Maßnahmen erreicht werden könnte, die die Wahrnehmung der Freiheitsrechte weniger beschränken würden (Haltern 2007, S. 751-757). Bei all diesen Bedingungen trägt der Mitgliedstaat die Beweislast, und die Anforderungen an die Erbringung eines (wissenschaftlichen) Beweises können, wie Dorte Martinsen (2009) gezeigt hat, in einem Maße erhöht werden, das ein negatives Ergebnis für den Mitgliedstaat geradezu garantiert.

Um die Asymmetrie zu verdeutlichen, lässt sich die Entscheidung gegen das ,VWGesetz" anführen, das für Entscheidungen in der Hauptversammlung eine Sperrminorität von 20 Prozent (statt der üblichen 25 Prozent) vorsah. ${ }^{36}$ Das Gericht sah in dieser Regel eine potentiell abschreckende Wirkung auf ausländische Direktinvestitionen. ${ }^{37}$ Beweisangebote der deutschen Seite, die zeigen sollten, in welchem Umfang VW-Aktien tatsächlich international gehandelt wurden und dass der Anteil ausländischer Direktinvestitionen so hoch war wie bei vergleichbaren Unternehmen, wurden für irrelevant erklärt. Mit anderen Worten, die Existenz eines Hemmnisses für den freien Kapitalverkehr wird in der Entscheidung als eine unwiderlegliche Vermutung behandelt. ${ }^{38}$

Ein anderes Beispiel ist wiederum die oben angeführte Österreich-Entscheidung, wo der EuGH immerhin den Gedanken erwog, dass die Gefahr einer Überfüllung der österreichischen Universitäten ein akzeptabler Rechtfertigungsgrund sein könnte. Dieser Gedanke wurde aber rasch mit der Begründung verworfen, dass dieses Problem sich auch durch nicht diskriminierende Aufnahmeprüfungen vermeiden ließe. ${ }^{39}$ Die Tatsache, dass Österreich seine eigenen Studenten bevorzugen müsste, um die im internationalen Vergleich niedrige Studierendenquote zu erhöhen und um eine ausreichende Anzahl von praktischen Ärzten für das österreichische Gesundheitswesen auszubilden, wurde von vornherein nicht als ein berechtigtes Argument anerkannt. ${ }^{40}$ In dieser asymmetrischen EuGH-Rechtsprechung sind europäische Rechte also substanziell und prozedural privi-

35 Rs. C-261/81, 10.11.1982, Tz. 12 (Rau).

36 Rs. C-74/07, 23.10.2007. Die Diskussion ist bei Tz. 55 ausgeführt.

37 Der EuGH konzedierte, dass private Anteilseigner eine Sperrminorität von 20 Prozent festlegen könnten, ohne gegen europäisches Recht zu verstoßen, aber beharrte darauf, dass dies einem demokratisch verantwortlichen Gesetzgeber nicht erlaubt sei.

38 Da nach der Dassonville-Formel ein potenzielles Hemmnis genügt, um als Verletzung der Freizügigkeitsrechte zu gelten, ist in der Tat schwer erkennbar, welche Art von Beweis diese Vermutung hätte widerlegen können.

39 Rs. C-147/03, Tz. 61.

40 In der Literatur wird gelegentlich behauptet, die österreichische Position sei vor dem EuGH nicht besonders kompetent vertreten worden. Aber derart offensichtliche Probleme hätten von einem fair abwägenden Gericht ex officio berücksicht werden müssen. 
legiert und genießen im Allgemeinen Vorrang vor sogar sehr wichtigen und politisch brisanten nationalen Belangen.

Eine dritte Asymmetrie folgt aus der Diskrepanz zwischen dem einheitlichen Europarecht und der Vielgestaltigkeit nationaler republikanischer Institutionen. Die auf dem EG-Vertrag basierenden wirtschaftlichen Grundfreiheiten werden selbstverständlich auf europäischer Ebene und ohne Berücksichtigung nationaler Unterschiede einheitlich definiert. Das Gleiche gilt dort, wo der EuGH andere subjektive Rechte auf europäischer Ebene anerkennt - Rechte, deren Zahl zunehmen wird, wenn der Lissabon-Vertrag in Kraft tritt. ${ }^{41}$ Sofern entgegenstehende nationale Belange überhaupt berücksichtigt werden, werden auch sie vom Gericht einheitlich (und äußerst restriktiv) definiert. Ein Beispiel ist die Laval-Entscheidung, die Mindestlöhne akzeptiert hätte, wenn sie durch ein staatliches Gesetz und nicht durch einen Kollektivvertrag festgelegt worden wären. Damit ignorierte das Gericht aber die Tatsache, dass staatliche Mindestlöhne zwar in vielen EU-Mitgliedstaaten üblich sind - aber eben nicht im ,neokorporatistischen“ Schweden. Dort, wo seit den 1930-er Jahren die Lohnbildung ausschließlich der kollektiven Verantwortung nationaler Gewerkschaften und Arbeitgeberverbände überlassen war, hätte ein staatliches Lohngesetz ein konstitutionelles Tabu verletzt (Edin u. Topel 1997).

Kurz gesagt, der vom EuGH etablierte rechtliche Rahmen, in dem die aus dem EG-Vertrag abgeleiteten und einheitlich definierten Individualrechte (in engen Grenzen) gegen die vom Gericht anerkannten und ebenso einheitlich definierten „Zwingenden Erfordernisse“ des nationalen öffentlichen Interesses abgewogen werden, ignoriert die Tatsache, dass einheitliches europäisches Recht Auswirkungen auf nationale Institutionen und Politikinhalte hat, die sich von Mitgliedstaat zu Mitgliedstaat stark unterscheiden. Solche Unterschiede existieren nicht nur bei den industriellen Beziehungen zwischen Arbeitgebern und Gewerkschaften, sondern auch bei den Unternehmensverfassungen, den öffentlichen Dienstleistungen, der öffentlichen Infrastruktur, der Medienpolitik, der Sozialpolitik, der Rentenpolitik, dem Gesundheitswesen oder der beruflichen und akademischen Bildung. Die gegenwärtig institutionalisierten Lösungen unterscheiden sich, weil sie aus historischen Konflikten und schwierigen Kompromissen zwischen konservativen, sozialdemokratischen und liberalen politischen Kräften hervorgegangen sind - weshalb auch jeder Versuch einer Veränderung überall hochbrisante politische Auseinandersetzungen provozieren muss.

Der politische Widerstand gegen Veränderungen wird dort am stärksten sein, wo Institutionen und Politik sich unmittelbar auf das Leben der Bürger auswirken. Dies ist am deutlichsten bei den Sozialtransfers und sozialen Dienstleistungen, bei den Beziehungen zwischen Arbeitgebern und Gewerkschaften, im Arbeitsrecht und im Bildungsund Gesundheitswesen. In vieler Hinsicht haben die überkommenen Lösungen hier den Status eines „Gesellschaftsvertrags“ erlangt, dessen Verbindlichkeit die Legitimität des nationalen Gemeinwesens unmittelbar berührt. Damit soll nicht gesagt werden, dass

41 Wie die Laval-Entscheidung jedoch deutlich machte, können die nichtwirtschaftlichen Grundrechte (einschließlich der Freiheit der Meinungsäußerung, der Versammlungsfreiheit und des Schutzes der Menschenwürde) nur unter der engen Beschränkung einer Proportionalitätsprüfung anerkannt werden, sobald sie mit im EG-Vertrag geschützten wirtschaftlichen Freiheitsrechten in Konflikt geraten (Rs. C-341/05, Tz. 94). 
solche normativ aufgewerteten Institutionen und Politiken gegen Veränderung immun sein sollten oder könnten. Tatsächlich ist ihre Überlebensfähigkeit unter zunehmenden äußeren und inneren Belastungen oft recht ungewiss (Scharpf u. Schmidt 2000). Aber wenn die Legitimität des nationalen Gemeinwesens erhalten bleiben soll, müssen solche Veränderungen in nationalen kommunikativen Diskursen erörtert, kritisiert und verteidigt werden - und zwar von Regierungen, die bereit sind, auch die Konsequenzen ihrer Politik gegenüber ihrer Wählern zu verantworten.

Tatsächlich anerkennt auch der Wortlaut des EG-Vertrages die Notwendigkeit, auf eben den genannten Politikfeldern die Autonomie der mitgliedstaatlichen Politik zu respektieren. In Maastricht und in Amsterdam wurden europäische Kompetenzen für Politikfelder, die für die Einzelstaaten von hoher normativer Relevanz sind, explizit ausgeschlossen. So sieht z.B. Artikel 137 Abs. 5 EG vor, dass die Zuständigkeit der Gemeinschaft auf dem Gebiet der Sozialpolitik ,nicht für das Arbeitsentgelt, das Koalitionsrecht, das Streikrecht sowie das Aussperrungsrecht" gelten soll. Ebenso sollen europäische Maßnahmen der Beschäftigungsförderung ,keinerlei Harmonisierung der Rechts- und Verwaltungsvorschriften der Mitgliedstaaten“ einschließen (Art. 129 Abs. 2 EG). Genau dieselbe Formulierung wird für die allgemeine Bildung (Art. 149 Abs. 4 EG), für die berufliche Bildung (Art. 150 Abs. 4) und für Kultur (Art. 152 Abs. 5 EG) wiederholt, während Art. 152 Abs. 5 EG vorsieht, dass: „die Gemeinschaft [...] die Verantwortung der Mitgliedstaaten für die Organisation des Gesundheitswesens und der medizinischen Versorgung in vollem Umfang respektiert.“ In anderen Bereichen hat der EG-Vertrag aus ähnlichen Gründen das Erfordernis eines einstimmigen Beschlusses des Rates beibehalten.

In dem vom Gerichtshof geschaffenen rechtlichen Rahmen können diese Verbote jedoch bestenfalls ${ }^{42}$ die politische Gesetzgebung auf europäischer Ebene beschränken. Für die richterliche Rechtsetzung werden sie dagegen als irrelevant angesehen, wo die Wahrnehmung nationaler Kompetenzen mit den Individualrechten des Gemeinschaftsrechts in Konflikt gerät. ${ }^{43}$ Das ist auch der Grund, weshalb die angeführten Entscheidungen ohne Rücksicht auf die vertraglichen Kompetenzschranken in der Lage waren,

42 Sollte die Kommission der Auffassung sein, dass fortbestehende Unterschiede zwischen den einzelstaatlichen Regeln (vorausgesetzt, diese haben jeweils die Verhältnismäßigkeitsprüfung bestanden) den Binnenmarkt beeinträchtigen oder den Wettbewerb verzerren, könnte jedoch auch in den angeführten Bereichen eine harmonisierende Richtlinie unter Art. 95 EG und Art. 96 Abs. 2 EG beschlossen werden (Haltern 2007, S. 740-741).

43 Die typische Formel lautet, dass die Mitgliedstaaten gewiss das Recht behalten, ihr eigenes Sozialversicherungs- und Gesundheitssystem zu gestalten, dabei aber selbstverständlich das Gemeinschaftsrecht beachten müssen, vgl. z.B. Rs. C-158/96, Tz. 16, 19-20 (Kohll). Dies verdeutlicht erneut die verfassungsstrukturelle Bedeutung der ursprünglich vom EuGH gewählten Begrifflichkeit: Indem er die Verpflichtungen des EG-Vertrages zur Schaffung eines gemeinsamen Marktes nicht nur als eine Quelle legislativer Kompetenzen behandelt, sondern auch als eine Garantie individueller Rechte interpretierte, hat er die rechtliche Möglichkeit beseitigt, Reservate nationaler Kompetenzen festzulegen, die nicht durch das Gemeinschaftsrecht erfasst werden können. Deshalb waren auch die Versuche der deutschen Länder, im Verfassungskonvent eine strikte Trennung europäischer und nationaler Kompetenzen zu erreichen, von vornherein aussichtslos. Ebenso wie in nationalen Bundesstaaten haben zentralstaatlich definierte und durchgesetzte Grundrechte auch in der EU eine zentralisierende Wirkung, die alle Politikfelder erreichen kann. Während legislative Kompetenzen durch Verfassungsänderungen begrenzt wer- 
Streiks in Finnland und in Schweden zu unterbinden, nationale Lohn- und Gehaltsregelungen in Deutschland und in Luxemburg auszuhebeln und nationale Regelungen für die Zulassung zum Hochschulstudium in Österreich ebenso zu beseitigen wie nationale Regelungen des Gesundheitswesens und der medizinischen Versorgung in Luxemburg und in den Niederlanden.

\section{Gesucht: Politische Schranken des Richterrechts}

Die neuere Rechtsprechung zeigt also: Selbst einstimmig beschlossene Vertragsänderungen, die von allen Mitgliedstaaten ratifiziert wurden, sind nicht in der Lage, die Autonomie der nationalen Politik gegen Interventionen des Gerichts schützen. Ohne ein eigenes politisches Mandat und im Widerspruch $\mathrm{zu}$ expliziten Vertragsbestimmungen, die die Reichweite des Europarechts begrenzen sollten, kann der Gerichtshof in Bereiche eingreifen, die für die demokratische Legitimität in den EU-Mitgliedstaaten eine kritische Bedeutung haben. Diese Entwicklung erscheint praktisch gefährlich und normativ nicht vertretbar.

Für die politische Praxis sind die Folgen dieser Rechtsprechung durchaus gefährlich: Die europäischen Sozialstaaten stehen unter dem Druck, sich an radikale Veränderungen der internationalen und innergesellschaftlichen Rahmenbedingungen anpassen zu müssen (Scharpf u. Schmidt 2000). Diese Anpassung muss jedoch durch legitimiertes politisches Handeln erreicht werden. Der Europäische Gerichtshof kann nationale Lösungen zwar beseitigen oder unterminieren, aber er kann selbst kein „soziales Europa“ schaffen. Gleichzeitig wird aber die europäische Gesetzgebung behindert durch die Verbote, die in den Verträgen von Maastricht und Amsterdam festgelegt wurden - und wenn diese aufgehoben würden, dann stünden hohe Konsensbarrieren und die existierende Divergenz nationaler Institutionen und normativer Präferenzen gemeinsamen europäischen Lösungen entgegen. Kurz: Das europäische Richterrecht untergräbt einzelstaatliche Lösungen, ohne dass Alternativen auf europäischer Ebene zur Verfügung stünden. Die praktische Folge ist ein Verlust politischer Problemlösungskapazität gerade auf den Politikfeldern, die für die Lebenswelt der Bürger und deren Legitimitätsbeziehung zu ihrem politischen Gemeinwesen von kritischer Bedeutung ist.

Das normative Problem hat seine Ursache in dem vom EuGH geschaffenen rechtlichen Rahmen, der keinen Raum lässt für eine angemessene Berücksichtigung der auf dem Spiel stehenden mitgliedstaatlichen Belange. Wo selbst minimale Einschränkungen des Gebrauchs europäischer Freiheitsrechte die nationalen Institutionen und Politiktraditionen außer Kraft setzen können, da kann ein normativ überzeugender Ausgleich zwischen den Erfordernissen der europäischen Kommunalität und dem legitimitätswichtigen Respekt für die demokratische Selbstbestimmung in den Mitgliedstaaten nicht einmal artikuliert werden. Aus dem gleichen Grunde können aber auch die juristischen Syllogismen, die diese Interventionen begründen sollen, in den kommunikativen Diskursen zwischen den Regierungen und ihren Wählern niemanden überzeugen. Kurz, die politisch

den können, gibt es gegen konstitutionell geschützte Individualrechte keine Kompetenzschranken. 
nicht verantwortete Ausdehnung des europäischen Richterrechts auf Bereiche, die für die politische Integrität der Mitgliedstaaten von kritischer Bedeutung sind, kann die Legitimationsgrundlagen des europäischen Mehrebenen-Gemeinwesens untergraben.

Die angemessene Konsequenz kann freilich weder die unbeschränkte Autonomie der Mitgliedstaaten noch eine Rückverlagerung der Kompetenz-Kompetenz auf die nationale Ebene sein (Weiler 1999a). Deren Ergebnis könnte in der Tat eine Eskalation protektionistischer und gemeinschaftsschädlicher nationaler Egoismen sein, die die Union zerreißen würde. Bei aller Kritik sollten wir auch sehen, dass es bei Viking und Laval - obwohl der Gerichtshof dies nie erwähnte - in der Tat auch um einen Verteilungskonflikt zwischen Hochlohn- und Niedriglohn-Ländern ging, dessen faire Regelung schwierige normative Fragen aufgeworfen hätte - und das Gleiche gilt vielleicht auch für Rüffert und Luxemburg. ${ }^{44}$ Es gibt deshalb gute normative Gründe für eine europäische Überprüfung nationaler Maßnahmen, die den freien Verkehr zwischen Mitgliedstaaten behindern. Aber eine solche Überprüfung müsste die faire Berücksichtigung aller auf dem Spiel stehenden Belange gewährleisten, die durch die Rechtsprechung des EuGH gerade ausgeschlossen wird. Deren selbstbezüglicher rechtlicher Rahmen verhindert jede Erörterung des normativen Spannungsverhältnisses zwischen den Werten der demokratischen Selbstbestimmung und einer Solidarität, die unter großen Anstrengungen auf nationaler Ebene erreicht wurde, und den ebenso gewichtigen Werten der der europäischen Kommunalität und der moralischen Verpflichtung zur „Einbeziehung der anderen“.

Aber welche Institution wäre besser als der Europäische Gerichtshof geeignet, die Balance zwischen legitimen (und divergierenden) nationalen Belangen auf der einen Seite und den gleichermaßen legitimen europäischen Belangen auf der anderen Seite herzustellen? Nach meiner Meinung käme dafür in erster Linie der Europäische Rat in Frage. ${ }^{45}$ Aus Sicht des betroffenen Mitgliedstaats wäre seine Entscheidung die einer Jury von Gleichrangigen, die die möglichen politischen Rückwirkungen einer Durchsetzung europäischen Richterrechts einschätzen können und wissen, dass sie sich selbst in derselben Lage wiederfinden könnten. Aber gleichzeitig wären die Regierungschefs als potentiell Betroffene auch höchst kritisch gegenüber einer Ausbeutung des gemeinsamen

44 Man sollte jedoch realistisch bleiben: Die transnationalen Umverteilungsvorteile (für Arbeitnehmer aus Niedriglohnländern), die sich eventuell aus diesen Urteilen ergeben könnten, werden durch inländische Umverteilungsnachteile in den Schatten gestellt, wenn die Konkurrenz das generelle Lohnniveau im Inland absenkt und zugleich staatliche Schutzgesetze und Kollektivvereinbarungen außer Kraft gesetzt werden.

45 Joseph Weiler (1999a, S. 322) forderte für die Entscheidung von Kompetenzfragen einen „Verfassungsrat" aus den Mitgliedern nationaler Verfassungsgerichte und ein ähnlicher Vorschlag wurde auch von Roman Herzog, dem früheren Präsidenten des Bundesverfassungsgerichts und des europäischen Grundrechts-Konvents unterstützt (Herzog u. Gerken 2008). Aus meiner Sicht wäre jedoch ein solcher Rat als eine rechtliche Instanz durch Präzedenzfälle gebunden und verpflichtet, seine Entscheidungsgründe allgemeingültig zu formulieren. Er würde deshalb wieder einheitliche Standards festlegen, die gerade nicht der legitimen Vielfalt der Institutionen und Praktiken der Mitgliedstaaten Rechnung tragen könnten. Nötig wäre statt dessen die disziplinierte „Adhocery“ eines politischen Gremiums, das versteht, dass es vorerst notwendig sein kann, das letzte Wort über Abtreibungen in Irland, Alkohol in Schweden oder Drogen in den Niederlanden (Kurzer 2001) den nationalen Parlamenten und Gerichten zu überlassen, selbst wenn dabei die Wahrnehmung anderswo geschützter europäischer Rechte behindert würde. 
Marktes durch protektionistische Trittbrettfahrer und gegenüber diskriminierenden und anderen gemeinschaftsschädlichen Praktiken. Vor allem aber wären die Mitglieder des Europäischen Rates in ihrer Rolle als „Herren der Verträge“ bestens legitimiert zu einem Urteil darüber, ob die Rechtsprechung die Grenzen einer vertretbaren Interpretation des europäischen Primär- und Sekundärrechtes so weit überschritten hat, dass eine politische Korrektur notwendig erscheint.

Aber selbst wenn die die diesem Vorschlag zugrundeliegenden Argumente überzeugen sollten, wäre seine Verwirklichung durch eine einstimmige Vertragsänderung höchst unwahrscheinlich. Es gäbe jedoch ein Szenario, das dies ändern könnte. Ich erinnere daran, was ich oben zur grundlegenden Abhängigkeit der Europäischen Union und ihres Rechtssystems von der freiwilligen Folgebereitschaft ihrer Mitgliedstaaten gesagt habe. Man stelle sich nun vor, die Regierungen einiger Mitgliedstaaten - z.B. Österreichs oder Schwedens oder Deutschlands - würden offen erklären, sie seien nicht bereit, einem bestimmten Urteil Folge zu leisten, weil sie darin eine vertragswidrige Intervention in einen der Autonomie der Mitgliedstaaten vorbehaltenen Bereich sehen. Wenn das alles wäre, würde die Erklärung eine Verfassungskrise auslösen. In der europäischen Praxis gibt es zwar viele Beispiele für die unvollständige Umsetzung und wohl auch für die stillschweigende Nichtbeachtung des Richterrechts, aber die offen erklärte Verweigerung der Folgebereitschaft würde an den Grundlagen des europäischen Rechtssystems rütteln. Aus diesem Grunde werden - und sollten - Regierungen auch zögern, diese ,nukleare Option“ ins Spiel zu bringen. Aber wie wäre es zu beurteilen, wenn die betroffene Regierung ihren begründeten Widerspruch gegen das Urteil als Antrag auf ein politisches Votum des Europäischen Rates formulierte und damit zugleich die verbindliche Zusage verbände, dass man dem Urteil auf jeden Fall folgen werde, sofern es durch eine Mehrheit des Rates bestätigt wird? ${ }^{46}$ Damit erschiene der Protest gegen eine EuGH-Entscheidung jedenfalls nicht mehr als grundsätzliche Aufkündigung der Loyalität gegenüber der Europäischen Union. ${ }^{47}$

$\mathrm{Ob}$ der Rat die Rolle akzeptieren würde, die ihm durch einen solchen Antrag zugemutet würde, ist natürlich höchst ungewiss. Aber wenn er sie akzeptierte, bekäme die Europäische Union endlich ein Forum ${ }^{48}$ und ein Verfahren, ${ }^{49}$ in dem das grundlegende

46 In seiner Logik ähnelt dieser Vorschlag dem Waiver-Verfahren in Art. IX Abs. 3 des WTOAbkommens, der es einem Mitgliedstaat gestattet, bei der Ministerkonferenz „unter außergewöhnlichen Umständen“ eine Ausnahme von geltendem WTO-Recht zu beantragen (Feichtner 2009). Eine gewisse Parallelität besteht auch zur Rolle des Rates im Defizitverfahren des Art. 104 EG oder im Art. 48 Abs. 2 des Lissabon-Vertrags.

47 Das impliziert auch, dass ,gute Europäer“ den automatischen (und oft vorauseilenden) Gehorsam gegenüber dem EuGH nicht länger als Loyalitätstest verwenden sollten. Sie haben gewiss jeden Grund, die politische Gesetzgebung und die politische Handlungsfähigkeit der Union zu stärken, aber daraus folgt nicht, dass sie die antidemokratischen Implikationen der „Integration durch Richterrecht" ignorieren müssten.

48 Praktisch müsste der Rat sich auf die Vorbereitungsarbeit eines ständigen Komitees stützen, das die relevanten Forderungen und Argumente anhören und würdigen würde. Die letzte Entscheidung sollte jedoch den Regierungschefs vorbehalten bleiben.

49 Meiner Meinung nach sollte die Bestätigung eines Urteils des EuGH nur eine einfache Mehrheit im Rat erfordern. 
Spannungsverhältnis zwischen den gleichermaßen legitimen Belangen der Gemeinschaft und der politischen Integrität der Mitgliedstaaten einen fairen Ausgleich finden könnte. ${ }^{50}$ Begrüßenswert wären auch die zu erwartenden Rückwirkungen auf die Rechtsprechung des Gerichtshofes. Schon die bloße Möglichkeit einer politischen Korrektur könnte das Gericht veranlassen, dem relativen Gewicht nationaler Belange im Verhältnis zu minimalen Behinderungen der europäischen Grundfreiheiten in der weiteren Entwicklung seiner rechtsdogmatischen Systematik mehr Aufmerksamkeit zuzuwenden. Wenn dies geschähe, dann wäre das Gemeinschaftsrecht - auch wenn ihm immer noch die „republikanische“ Input-Legitimität fehlte - nicht mehr als ein ausschließlich auf die Maximierung eines gemeinschaftsblinden „Individualismus“ (Somek 2008) gerichtetes Normensystem zu beschreiben.

\section{Literatur}

Alter, Karen. 2001. Establishing the supremacy of European law. Oxford: Oxford University Press.

Anderson, Christopher J., Andrè Blais, Shaun Bowler, Todd Donovan, and Ola Listhaug. 2005. Losers' consent: Elections and democratic legitimacy. Oxford: Oxford University Press.

Aristoteles. 1989. Politik: Schriften zur Staatstheorie. Translated and edited by Franz F Schwarz. Stuttgart: Reclam.

Bartolini, Stefano. 2005. Restructuring Europe: Centre formation, system building, and political restructuring between the nation State and the European Union. Oxford: Oxford University Press.

Bartolini, Stefano. 2008. Taking 'constitutionalism' and 'legitimacy' seriously. European Governance Papers, Discussion Paper 1-2008. Florence: Robert Schuman Centre for Advanced Study, European University Institute. http://www.connex-network.org/eurogov/pdf/discuss paper_01_2008.pdf.

Battigalli, Pierpaolo, and Giovanni Maggi. 2003. International agreements on product standards: An incomplete-contracting theory. Working Paper 9533. Cambridge: National Bureau of Economic Research. http://www.nber.org/papers/w9533.

Beetham, David. 1991. The legitimation of power. Basingstoke: Macmillan.

Bellamy, Richard. 2007. Political constitutionalism: A republican defence of the constitutionality.of democracy. Cambridge: Cambridge University Press.

Berlin, Isaiah. 1958. Two concepts of liberty: An inaugural lecture. Oxford: Clarendon Press.

Bickel, Alexander. 1962. The least dangerous branch: The supreme court at the bar of politics. Indianapolis: Bobbs-Merrill.

Börzel, Tanja A., and Meike Dudziak. 2007. Why member states do (not) comply with European law. CES Working Paper 148. Cambridge: Harvard University.

Buchanan, James M., and Gordon Tullock. 1962. The calculus of consent: Logical foundations of constitutional democracy. Ann Arbor: University of Michigan Press.

50 Einmal eingeführt, könnte ein solches Verfahren auch zur Erlaubnis von „,conditional opt-outs“ vom gemeinschaftsrechtlichen acquis eingesetzt werden. Dies würde die Lösung von Problemen erleichtern, die durch das quasi-irreversible Sekundärrecht verursacht werden. Die Möglichkeit späterer Opt-outs könnte überdies die politische Einigung über neue Gesetze begünstigen. Eine ähnliche Lösung wurde im Zusammenhang mit der Föderalismusreform in Deutschland diskutiert (Scharpf 2008, 2009). Aber diese Erweiterungen gehen über das hier vertretene Argument hinaus, und ihre Diskussion würde die Grenzen dieses Beitrags sprengen. 
Burkhart, Simone. 2008. Blockierte Politik: Ursachen und Folgen von „Divided government“ in Deutschland. Frankfurt a.M.: Campus.

Caporaso, James A. 2000. The European Union: Dilemmas of regional integration. Boulder: Westview.

Caporaso, James A. and Sidney Tarrow. 2008. Polanyi in Brussels: European institutions and the embedding of markets in society. Conference paper. American Political Science Association Meeting, Boston, August 28, 2008.

Cappelletti, Mauro, Monica Seccombe, and Joseph H. H Weiler (gen. eds.). 1985a. Integration through law: Europe and the American federal experience. Berlin: De Gruyter.

Cappelletti, Mauro, Monica Seccombe, and Joseph H.H Weiler. 1985b. A general Iitroduction. In Integration through law: Europe and the American federal experience, ed. Mauro Cappelletti, Monica Seccombe and Joseph H.H Weiler, 1-68. Berlin: De Gruyter.

Cicero, Marcus Tullius. 1995. De re publica. Translated and edited by Karl Büchner. Stuttgart: Reclam.

Cichowski, Rachel A. 2004. Women's Rights, the European Court, and Supranational Constitutionalis. In Law \& Society Review 38 (3): 489-512.

Dahl, Robert A. 1967. Pluralist democracy in the United States: Conflict and consent. Chicago: Rand McNally.

Dahl, Robert. 1989. Democracy and its critics. New Haven: Yale University Press.

Dryzek, John S. 2000. Deliberative democracy and beyond: Liberals, critics, contestations. Oxford: Oxford University Press.

Easton, David.1965. A systems analysis of political life. New York: John Wiley.

Edin, Per-Anders, and Robert Topel. 1997. Wage policy and restructuring: The Swedish labor market since 1960. In The welfare state in transition: Reforming the Swedish model, ed. Richard B. Freeman, Robert Topel and Birgitta Swedenborg, 155-201. Chicago: University of Chicago Press.

Egle, Christoph, und Reimut Zohlhöfer, Hrsg. 2007. Ende des rot-grünen Projekts: Eine Bilanz der Regierung Schröder 2002-2005. Wiesbaden: VS-Verlag.

Elster, Jon. 1998. Introduction. In Deliberative Democracy, ed. Jon Elster, 1-18. Cambridge: Cambridge University Press.

Falkner, Gerda, Oliver Treib, Miriam Hartlapp, and Simone Leiber. 2005. Complying with Europe: EU harmonisation and soft law in the member states. Cambridge: Cambridge University Press.

Feichtner, Isabel. 2009. The waiver-power of the WTO. Opening the WTO for political deliberation on the reconciliation of public interests. MS. Heidelberg: Max-Planck-Institut für ausländisches öffentliches Recht und Völkerrecht.

Ferrera, Maurizio. 2005. The boundaries of welfare: European integration and the new spatial politics of social protection. Oxford: Oxford University Press.

Fisher, Louis. 1988. Constitutional dialogues: Interpretation as political process. Princeton: Princeton University Press.

Follesdal, Andreas, and Simon Hix. 2006. Why there is a democratic deficit in the EU: A response to Majone and Moravesik. Journal of Common Market Studies 44 (3): 533-562.

Ganghof, Steffen, and Philipp Genschel. 2008a. Taxation and democracy in the EU. Journal of European Public Policy 15 (1): 58-77.

Ganghoff, Steffen, and Philipp Genschel. 2008b. Deregulierte Steuerpolitik: Körperschaftsteuerwettbewerb und Einkommensbesteuerung in Europa. In Die politische Ökonomie der europäischen Integration, Hrsg. Martin Höpner und Armin Schäfer, 311-334. Frankfurt a.M.: Campus.

Garrett, Geoffrey. 1992. International cooperation and institutional choice: The European community's internal market. International Organization 46: 533-560. 
Garrett, Geoffrey. 1995. The politics of legal integration in the European Union. International Organization 49 (1): 171-181.

Genschel, Philipp, Achim Kemmerling, and Eric Seils. 2008. Accelerating downhill: How the EU shapes corporate tax competition in the single market. Unpublished manuscript. Bremen: Jacobs University.

Greven, Michael T. 2000. Can the European Union finally become a democracy? In Democracy beyond the state? The European dilemma and the emerging global order, ed. Michael T. Greven and Louis W. Pauly, 35-62. Lanham: Rowman \& Littlefield.

Habermas, Jürgen. 1962. Strukturwandel der Öffentlichkeit: Untersuchungen zu einer Kategorie der bürgerlichen Gesellschaft. Neuwied: Luchterhand.

Habermas, Jürgen. 1992. Faktizität und Geltung: Beiträge zur Diskurstheorie des Rechts und des demokratischen Rechtsstaats. Frankfurt a.M.: Suhrkamp.

Habermas, Jürgen. 1996. Die Einbeziehung des Anderen: Studien zur politischen Theorie. Frankfurt a.M.: Suhrkamp.

Habermas, Jürgen. 2008. Hat die Demokratie noch eine epistemische Dimension? Empirische Forschung und normative Theorie. In Ach Europa: Kleine politische Schriften XI., Hrsg. Jürgen Habermas, 138-191. Frankfurt a.M.: Suhrkamp.

Hall, Peter A., and David Soskice, eds. 2001. Varieties of Capitalism: The Institutional Foundations of Comparative Advantage. Oxford: Oxford University Press.

Haltern, Ulrich. 2007. Europarecht: Dogmatik im Kontext. 2. Aufl. Tübingen: Mohr Siebeck.

Harlow, Carol. 2002. Accountability in the European Union. Oxford: Oxford University Press.

Hemerijck, Anton, Brigitte Unger, und Jelle Visser. 2000. How small countries negotiate change: Twenty-Five years of policy adjustment in Austria, the Netherlands, and Belgium. In Welfare and work in the open economy, Vol. 2: Diverse responses to common challenges, ed. Fritz. W. Scharpf and Vivien A.Schmidt, 175-263. Oxford: Oxford University Press.

Héritier, Adrienne. 2003. New modes of governance in Europe: Increasing political capacity and policy effectiveness? In The state of the European Union, Vol. 6: Law, politics and society, ed. Tanja A. Boerzel and Rachel A. Cichowski, 105-126.Oxford: Oxford University Press.

Héritier, Adrienne, and Dirk Lehmkuhl. 2008. Introduction: The shadow of hierarchy and new modes of governance. Journal of Public Policy 28 (1): 1-17.

Herzog, Roman, und Lüder Gerken. 2008. Stoppt den Europäischen Gerichtshof. Frankfurter Allgemeine Zeitung, 8. September.

Hix, Simon. 2008. What's wrong with the European Union and how to fix it. Cambridge: Polity.

Hobbes, Thomas. 1986 [1651]. Leviathan:Or the matter, forme and power of a commonwealth ecclesiasticall and civil. New York: Collier Books.

Höffe, Otfried. 2002. Demokratie im Zeitalter der Globalisierung. Revidierte Auflage. München: C.H. Beck.

Höpner, Martin, und Armin Schäfer. 2007. A new phase of European integration: Organized capitalism in post-Ricardian Europe. MPIfG Discussion Paper 07/4. Cologne: Max Planck Institute for the Study of Societies.

Höreth, Marcus. 2008. Die Selbstautorisierung des Agenten: Der Europäische Gerichtshof im Vergleich zum U.S. Supreme Court. Baden-Baden: Nomos.

Joerges, Christian, and Navraj Singh Ghaleigh, eds. 2003. Darker legacies of law in Europe: The shadow of national socialism and fascism over Europe and its legal traditions. Oxford: Hart Publishing.

Kant, Immanuel. 1961 [1785]. Grundlegung zur Metaphysik der Sitten. Stuttgart: Reclam.

Kant, Immanuel. 1966 [1797]. Metaphysik der Sitten: Einleitung in die Rechtslehre. Hamburg: Felix Meiner.

Kant, Immanuel. 1992 [1793]. Über den Gemeinspruch: Das mag in der Theorie richtig sein, taugt aber nicht für die Praxis. Hamburg: Felix Meiner. 
Kohler-Koch, Beate, und Berthold Rittberger. 2006. Review Article: The 'governance turn' in EU Studies. Journal of Common Market Studies 44 (s1): 27-49.

Kramer, Larry D. 2004. The people themselves: Popular constitutionalism and judicial review. Oxford: Oxford University Press.

Kurzer, Paulette. 2001. Markets and moral regulation: Cultural changes in the European Union. Cambridge: Cambridge University Press.

Lewis, Jeffrey. 2005. The Janus face of Brussels: Socialization and everyday decision making in the European Union. International Organization 59 (4): 937-971.

Lübbe-Wolff, Gertrude. 2007. Expropriation der Jurisprudenz? In Das Proprium der Rechtswissenschaft, Hrsg. Christoph Engel und Wolfgang Schön, 282-292. Tübingen: Mohr Siebeck.

Machiavelli, Niccolò. 1966 [1531]. Discorsi: Gedanken über Politik und Staatsführung. Stuttgart: Kröner.

MacIntyre, Alasdair. 1984. After Virtue. Notre Dame: University of Notre Dame Press.

MacIntyre, Alasdair. 1988. Whose justice? Which rationality? Notre Dame: University of Notre Dame Press.

Mair, Peter. 2008. Popular democracy and the European Union polity. In Meaning and practice of accountability in the EU multi-level context, ed. Deirdre Curtin and Anchrit Wille, 19-62. Connex Report Series, Vol. 7. Mannheim: University of Mannheim.

Majone, Giandomenico. 1996. Regulatory legitimacy. In Regulating Europe, ed. Giandomenico Majone, 284-301. London: Routledge.

Majone, Giandomenico. 1998. Europe's 'democratic deficit': The question of standards. European Law Journal 4 (1): 5-28.

March, James G., and Johan P. Olsen. 1989. Rediscovering institutions: The organizational basis of institutions. New York: Free Press.

Martinsen, Dorte. 2005. The Europeanization of welfare: The domestic impact of intra-European social security. Journal of Common Market Studies 43 (5): 1027-1054.

Martinsen, Dorte. 2009. Extending health care regulation in the European Union: Through law and evidence. West European Politics 32 (4): forthcoming.

Martinsen, Dorte, and Karsten Vrangbaek. 2008. The Europeanization of health care governance: Implementing the market imperatives of Europe. Public Administration 86 (1): 169-184.

Maskin, Eric, and Jean Tirole. 1999. Unforeseen contingencies and incomplete contracts. Review of Economic Studies 66 (1): 83-114.

Mény, Yves, and Yves Surel, eds. 2002. Democracies and the populist challenge. Houndsmill: Palgrave Macmillan.

Michelman, Frank I. 1989. Conceptions of democracy in American constitutional argument: The case of pornography regulation. Tennessee Law Review 56 (2): 203-304.

Möllers, Christoph. 2008. Die drei Gewalten: Legitimation der Gewaltengliederung in Verfassungsstaat, Europäischer Integration und Internationalisierung. Göttingen: Velbrück Wissenschaft.

Moravcsik, Andrew. 1998. The choice for Europe: Social purpose and state power from Messina to Maastricht. Ithaca: Cornell University Press.

Moravcsik, Andrew, and Andrea Sangiovanni. 2003. On democracy and 'public interest' in the European integration. In Die Reformierbarkeit der Demokratie: Innovationen und Blockaden, ed. Renate Mayntz and Wolfgang Streeck, 122-150. Frankfurt a.M.: Campus.

Münch, Richard. 2008a. Die Konstruktion der europäischen Gesellschaft: Zur Dialektik von transnationaler Integration und nationaler Desintegration. Frankfurt a.M.: Campus.

Münch, Richard. 2008b. Constructing a European society by jurisdiction. European Law Journal 14 (5): 519-541.

Nicolaidis, Kalypso, and Robert Howse, eds. 2001. The federal vision: Legitimacy and levels of governance in the United States and the European Union. Oxford: Oxford University Press. 
Nicolaïdis, Kalypso, and Susanne K. Schmidt. 2007. Mutual recognition 'on trial': The long road to services liberalization. Journal of European Public Policy 14 (5): 717-734.

Pateman, Carole. 1985. The problem of political obligation: A critique of liberal theory. Berkeley: University of California Press.

Pitkin, Hanna Fenichel. 1981. Justice: On relating private and public. Political Theory 9 (3): $327-352$.

Pocock, John G.A. 1975. The Machiavellian moment: Florentine political thought and the Atlantic republican tradition. Princeton: Princeton University Press.

Pollak, Johannes. 2008. Ist eine europäische Identität möglich? Warum wir lernen müssen, Zwiebeln zu lieben. In 'Schmerzliche Erfahrungen der Vergangenheit' und der Prozess der Konstitutionalisierung Europas, Hrsg. Christian Joerges, Matthias Mahlmann und Ulrich K. Preuß, 63-80. Wiesbaden: VS-Verlag.

Preuss, Ulrich K. 1999. National, Supranational and International Solidarity. In Solidarity: philosophical studies in contemporary culture 5, ed. Kurt Bayertz, 281-292. Dordrecht: Kluwer.

Rousseau, Jean-Jacques. 1959 [1762]. Der Gesellschaftsvertrag: Oder die Grundsätze des Staatsrechts. Translated by H. Denhardt. Stuttgart: Reclam, 1769/70.

Scharpf, Fritz W. 1988. The joint decision trap: Lessons from German federalism and European integration. Public Administration 66 (3): 239-278.

Scharpf, Fritz. 1997. Games real actors play: Actor-centered institutionalism in policy research. Boulder: Westview.

Scharpf, Fritz. 1999. Governing in Europe: Effective and democratic? Oxford: Oxford University Press.

Scharpf, Fritz. 2001. Notes toward a theory of multilevel governing in Europe. Scandinavian Political Studies 24 (1): 1-26.

Scharpf, Fritz. 2008. Community, diversity and autonomy: The challenges of reforming German federalism. German Politics 17 (4): 509-521.

Scharpf, Fritz. 2009. Föderalismusreform. Kein Ausweg aus der Politikverflechtungsfalle? Frankfurt a.M: Campus.

Scharpf, Fritz W., and Vivien A Schmidt, eds. 2000. Welfare and work in the open economy. 2 vols. Oxford: Oxford University Press.

Schmidt, Susanne K. 2007. Mutual recognition as a new mode of governance. Journal of European Public Policy 14 (5): 667-681.

Schmidt, Vivien A. 2004. The European Union: Democratic legitimacy in a regional state. Journal of Common Market Studies 42 (4): 975-999.

Schmidt, Vivien A. 2006. Democracy in Europe: The EU and national politics. Oxford: Oxford University Press.

Skinner, Quentin. 1998. Liberty before liberalism. Cambridge: Cambridge University Press.

Somek, Alexander. 2008. Individualism: An essay on the authority of the European Union. Oxford: Oxford University Press.

Taylor, Charles. 1992. The ethics of authenticity. Cambridge: Harvard University Press.

Tsebelis, George. 2002. Veto players: How political institutions work. Princeton: Princeton University Press.

Vreese, Claes de, and Hermann Schmitt, eds. 2007. A European public sphere: How much of it do we have and how much do we need? Connex Report Series, Vol. 2. Mannheim: University of Mannheim.

Weaver,R Kent. 1986. The politics of blame avoidance. Journal of Public Policy 6 (4): 371-398.

Weiler, Joseph H.H. 1999a. The autonomy of the community legal order: Through a looking glass. In The Constitution of Europe, ed. Joseph. H.H. Weiler, 286-323. Cambridge: Cambridge University Press.

Weiler, Joseph H.H. 1999b. To be a European citizen: Eros and civilization. In The Constitution of Europe, by J.H.H. Weiler, 324-357. Cambridge: Cambridge University Press. 
Weiler, Joseph H.H. 2003. In defence of the status quo. Europe's constitutional 'Sonderweg'. In European constitutionalism beyond the state, ed. J.H.H. Weiler and Marlene Wind, 7-23. Cambridge: Cambridge University Press.

Weiler, Joseph H.H., and Nicholas J.S Lockhart. 1995. 'Taking rights seriously': The European court and its fundamental rights jurisprudence. Common Market Law Review 32 (1): 51-94 and (3): 579-627.

Wind, Marlene, Dorte Sindbjerg Martinsen, and Gabriel Pons Rotger. 2009. The uneven legal push for Europe. Questioning variation when national courts go to Europe. European Union Politics 10 (1): 63-88.

Wollenschläger, Ferdinand. 2007. Grundfreiheit ohne Markt: Die Herausbildung der Unionsbürgerschaft im unionsrechtlichen Freizügigkeitsregime. Tübingen: Mohr Siebeck.

Zürn, Michael, and Christian Joerges, eds. 2005: Law and governance in postnational Europe: Compliance beyond the nation-State. Cambridge: Cambridge University Press. 